\title{
Complex roles for reactive astrocytes in the triple transgenic mouse model of Alzheimer disease
}

Océane Guillemaud $^{\mathrm{a}, \mathrm{b} \#}$, Kelly Ceyzériat ${ }^{\mathrm{a}, \mathrm{b} \# 1}$, Thomas Saint-Georges ${ }^{\mathrm{a}, \mathrm{b}}$, Karine Cambon ${ }^{\mathrm{a}, \mathrm{b}}$, Fanny Petit $^{\mathrm{a}, \mathrm{b}}$, Lucile Ben Haim ${ }^{\mathrm{a}, \mathrm{b}}$, Maria-Angeles Carrillo-de Sauvage ${ }^{\mathrm{a}, \mathrm{b}}$, Martine Guillermier ${ }^{\mathrm{a}, \mathrm{b}}$, Sueva Bernier $^{\mathrm{a}, \mathrm{b}}$, Anne-Sophie Hérard ${ }^{\mathrm{a}, \mathrm{b}}$, Charlène Joséphine ${ }^{\mathrm{a}, \mathrm{b}}$, Alexis Pierre Bémelmans $\mathrm{s}^{\mathrm{a}, \mathrm{b}}$, Emmanuel Brouillet $^{\mathrm{a}, \mathrm{b}}$, Philippe Hantraye ${ }^{\mathrm{a}, \mathrm{b}}$, Gilles Bonvento ${ }^{\mathrm{a}, \mathrm{b}}$, Carole Escartin ${ }^{\mathrm{a}, \mathrm{b}}$

\footnotetext{
${ }^{a}$ Commissariat à l'Energie Atomique et aux Energies Alternatives, Département de la Recherche Fondamentale, Institut de Biologie François Jacob, MIRCen, 18 route du panorama, F-92260 Fontenay-aux-Roses, France

${ }^{\mathrm{b}}$ Centre National de la Recherche Scientifique, Université Paris-Sud, Université Paris-Saclay, UMR 9199, Neurodegenerative Disease Laboratory, 18 route du panorama, F-92260 Fontenay-aux-Roses, France
}

\footnotetext{
\# equal contribution

${ }^{1}$ Present address: Division of Adult Psychiatry, Department of Psychiatry, University Hospitals of Geneva and

Division of Nuclear medicine, University Hospitals of Geneva and Geneva University, Geneva, Switzerland.
}

\section{Corresponding author:}

Carole Escartin

UMR9199

MIRCen

18, route du Panorama

92260 Fontenay-aux-roses

France

Carole.escartin@cea.fr

Declarations of interest: none. 


\section{ABSTRACT}

In Alzheimer disease (AD), astrocytes undergo complex changes and become reactive. The consequences of this reaction are still unclear. To evaluate the net impact of reactive astrocytes in $\mathrm{AD}$, we recently developed viral vectors targeting astrocytes that either activate or inhibit the JAK2STAT3 pathway, a central cascade controlling astrocyte reaction.

We aimed to evaluate whether reactive astrocytes contribute to Tau as well as amyloid pathologies in the hippocampus of 3xTg-AD mice, an AD model that develops Tau hyper-phosphorylation and aggregation in addition to amyloid deposition. JAK2-STAT3 pathway-mediated modulation of reactive astrocytes in $25 \%$ of the hippocampus of $3 \times \mathrm{Tg}-\mathrm{AD}$ mice, did not significantly influence Tau phosphorylation or amyloid processing and deposition at early, advanced and terminal stage of the disease. Interestingly, inhibition of the JAK2-STAT3 pathway in hippocampal astrocytes did not improve short-term spatial memory in the Y maze but it did reduce anxiety in the elevated plus maze. Our unique approach to specifically manipulate reactive astrocytes in situ show these cells may impact behavioral outcomes without influencing Tau or amyloid pathology.

Key words: Reactive astrocytes; Alzheimer disease; Tau; JAK-STAT3 pathway; SOCS3; Neuroinflammation. 


\section{INTRODUCTION}

Alzheimer disease (AD) is a devastating neurodegenerative disease and the most common form of dementia (Querfurth and LaFerla, 2010). It is characterized by extracellular accumulation of amyloid plaques, Tau hyper-phosphorylation, synaptic alterations and neuronal degeneration. The exact mechanisms responsible for AD are still disputed (Sala Frigerio and De Strooper, 2016). The amyloid cascade hypothesis was first to be put forward. Here, successive cleavages of the amyloid precursor protein (APP) by $\beta$ - and $\gamma$-secretases, generate high levels of soluble amyloid beta (A $\beta$ ) peptides. These peptides then oligomerize and form amyloid plaques in the brain of $\mathrm{AD}$ patients and can cause synaptic dysfunction, neurodegeneration and cognitive impairment (Hardy and Higgins, 1992). The amyloid hypothesis was later refined to include Tau hyper-phosphorylation as a key pathogenic element (Kametani and Hasegawa, 2018; Medina and Avila, 2014). Hyper-phosphorylated Tau proteins can also oligomerize and form neurofibrillary tangles that inhibit microtubule assembly and alter neuronal functions. Indeed, progression of Tau pathology from the entorhinal cortex to other isocortex regions (Braak and Braak, 1991) correlates better than amyloid pathology with cognitive impairment observed in patients (Arriagada et al., 1992; Masters et al., 2015).

Recently, evidence has emerged showing that $\mathrm{AD}$ is not only caused by cell-autonomous processes within neurons, and that glial cells may also play an instrumental role in disease progression (Arranz

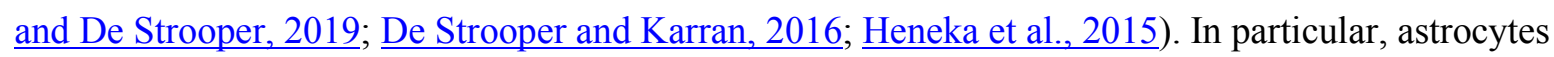
perform a number of key brain functions, including neurotransmitter recycling, ion homeostasis, metabolic and trophic support (Verkhratsky and Nedergaard, 2018). In the brain of AD patients or in animal models, astrocytes are reactive and participate in neuroinflammation. Reactive astrocytes are classically characterized by morphological changes (hypertrophy and process reorganization), as well as upregulation of intermediate filament proteins [Glial fibrillary acidic protein (GFAP), vimentin, (Hol and Pekny, 2015)]. Much less is known about their functional features and exactly how they impact AD outcomes (Ben Haim et al., 2015a). Several studies have reported alterations in some of the multiple functions operated by astrocytes such as glutamate uptake (Masliah et al., 1996), metabolic supply (Allaman et al., 2010; Sancheti et al., 2014), production of antioxidant molecules 
(Abeti et al., 2011; Allaman et al., 2010) or regulation of synaptic transmission (Delekate et al., 2014;

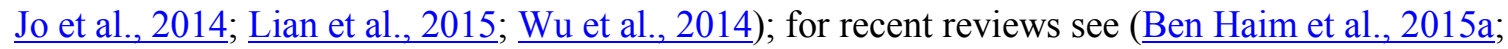
Chun and Lee, 2018). Interestingly, astrocytes may directly participate in APP metabolism and A $\beta$ clearance through various mechanisms, ranging from extracellular cleavage to phagocytosis or drainage through the glymphatic system (Ries and Sastre, 2016). But how these clearance mechanisms are altered when astrocytes acquire reactive features during $\mathrm{AD}$ progression is still unknown.

Similarly, the exact impact of reactive astrocytes on Tau hyper-phosphorylation and aggregation remains to be established (Kahlson and Colodner, 2015; Leyns and Holtzman, 2017).

We recently developed a method that efficiently blocks morphological and molecular changes in reactive astrocytes, by inhibiting the Janus kinase -Signal transducer and activator of transcription 3 (JAK-STAT3) pathway, through astrocyte-specific expression of its inhibitor suppressor of cytokine signaling 3 [SOCS3, (Ceyzeriat et al., 2018)]. We showed that reactive astrocytes promote amyloid deposition and learning deficits in the APP/PS1dE9 transgenic mouse model of AD, and early synaptic deficits in 3xTg-AD mice, another transgenic model that also develops Tau pathology.

Here, we aimed to evaluate in $3 \times \mathrm{Tg}-\mathrm{AD}$ mice, whether reactive astrocytes 1) influence Tau and amyloid pathologies at different disease stages and 2) have long-term effects on cognitive functions. Unexpectedly, we found that JAK2-STAT3 activation in reactive astrocytes does not affect Tau or amyloid pathology in 3xTg-AD mice at early, advanced and terminal disease stages. In addition, inhibition of reactive astrocytes does not improve working memory but it does reduce anxiety in 3xTg-AD mice.

\section{MATERIAL AND METHODS}

\section{Animals.}

Triple transgenic AD (here referred to as 3xTg mice) mice express human APPswe and human $\mathrm{Tau}^{\mathrm{P} 301 \mathrm{~L}}$ under a Thy-1 promoter as well as a point mutation on the mouse Psenl gene (PS1 ${ }^{\mathrm{M} 146 \mathrm{~V}}$ ), on a mixed C57BL/6J x 129Sv background (Oddo et al., 2003). C57BL/6J x 129Sv mice were used as controls. Only females were used in this study, as they display earlier neuropathology (Carroll et al., 2010; Hirata-Fukae et al., 2008). Breeding pairs were obtained from the Mutant Mouse Regional 
Resource Centers. All experimental protocols were approved by a local ethics committee (CETEA $\mathrm{N}^{\circ} 44$ ) and submitted to the French Ministry of Education and Research (Approvals \# APAFIS\#45652016031711426915 v3 and APAFIS\#4503-2016031409023019). They were performed in an authorized facility (\#D92-032-02), under the supervision of a veterinarian and in strict accordance with the recommendations of the European Union (2010-63/EEC) for the care and use of laboratory animals. All efforts were made to promote animal welfare. Mice were housed under standard environmental conditions (12-hour light-dark cycle, temperature: $22 \pm 1{ }^{\circ} \mathrm{C}$ and humidity: $50 \%$ ) with ad libitum access to food and water. Mice of the appropriate genotype were randomly allocated to experimental groups.

\section{Stereotactic injections of viral vectors.}

Adeno-associated viruses of serotype 9 (AAV2/9) encoding Gfp, murine Socs3, or a constitutively active form of Jak2 (JAK2 ${ }^{\mathrm{T} 875 \mathrm{~N}}$, aka JAK2ca) were produced and titrated at the MIRCen viral vector facility, as described previously (Ceyzeriat et al., 2018). All transgenes were under the control of the gfa $\mathrm{ABC}_{1} \mathrm{D}$ promoter, which drives specific expression in astrocytes, including those of the dorsal hippocampus, where the viral vectors were delivered [(Ceyzeriat et al., 2018), Fig 1a, Supplemental Fig. 2].

Before surgery, mice were anesthetized with a mixture of ketamine $(100 \mathrm{mg} / \mathrm{kg})$ and xylazine $(10$ $\mathrm{mg} / \mathrm{kg})$ and received s.c. injection of lidocaine $(7 \mathrm{mg} / \mathrm{kg})$ at the incision site, $10 \mathrm{~min}$ before injection. Mice received paracetamol in drinking water $(1.6 \mathrm{mg} / \mathrm{ml})$ for $48 \mathrm{~h}$ after surgery. AAV were diluted in $0.1 \mathrm{M}$ phosphate buffer saline (PBS) with $0.001 \%$ pluronic acid, at a final total concentration of 2.5 $10^{9}$ viral genome $/ \mu 1$. Mice were positioned on a stereotactic apparatus with tooth bar set at $0 \mathrm{~mm}$. They were injected in the CA1 region of the hippocampus (coordinates: $-3 \mathrm{~mm}$ antero-posterior, $+/-3$ mm lateral and $-1.5 \mathrm{~mm}$ ventral, from dura) with $2 \mu 1$ of AAV suspensions at a rate of $0.2 \mu 1 / \mathrm{min}$. After $5 \mathrm{~min}$, the cannula was slowly removed and the skin sutured. Mice recovered for at least 2 months before analysis. 


\section{Experimental groups.}

Four mouse cohorts were analyzed (Supplemental Fig. 1). The number of mice analyzed is mentioned in each figure.

1. Homozygous $3 x T g$ mice were injected at 6 months with AAV-GFP (3xTg-GFP mice) or AAVSOCS3 + AAV-GFP at the same total viral titer (3xTg-SOCS3 mice), to block reactive astrocytes. AAV-GFP was co-injected with AAV-SOCS3 to visualize infected cells. WT mice of the same age, gender and genetic background were injected with AAV-GFP (WT-GFP) as control. Mice were tested on the elevated plus maze and the Y maze at 8 and 14-15 months. Mice were euthanized by an overdose of pentobarbital at the age of 16 months. The two brain hemispheres were rapidly dissected on ice. One was drop-fixed in 4\% paraformaldehyde (PFA) and used for immunohistochemistry. The other was cut into 1-mm-thick slices on ice and the hippocampal $\mathrm{GFP}^{+}$region was dissected out under a fluorescent macroscope, snap frozen in liquid nitrogen and stored at $-80^{\circ} \mathrm{C}$ until protein extraction. 2. At 3 months, WT mice were injected with AAV-GFP, and 3xTg mice were injected with AAVGFP or with AAV-JAK2ca + AAV-GFP (same total viral titer, 3xTg-JAK2ca mice), to exacerbate astrocyte reaction. Mice were euthanized as 16 months and analyzed by histological and biochemical approaches as described for the first cohort.

3. At 4 months, WT mice were injected with AAV-GFP, and 3xTg mice were injected with AAVGFP or with AAV-SOCS3 + AAV-GFP. Mice were euthanized by an overdose of pentobarbital at the age of 9 months. The hippocampus was dissected out, snap frozen in liquid nitrogen and stored at $80^{\circ} \mathrm{C}$ until protein extraction.

4. At 24 months, WT mice were injected with AAV-GFP, and 3xTg mice were injected with AAVGFP or with AAV-SOCS3 + AAV-GFP. Mice were analyzed by biochemical approaches at 26 months as described for cohort 3 .

The early and mid-stage cohorts (cohorts 1,2,3) were all injected at an early stage (3 to 6 months), when there is no detectable amyloid or Tau deposition (Carroll et al., 2010; Oddo et al., 2003). Endstage mice were injected at 24 months to assess whether a late manipulation of the JAK-STAT3 pathway in astrocytes could reverse or reduce advanced amyloid and Tau pathology. 


\section{Immunohistology.}

Dedicated hemispheres were post-fixed for $24 \mathrm{~h}$ in $4 \%$ PFA, cryoprotected in a 30\% sucrose solution and cut on a freezing microtome into serial $30-\mu \mathrm{m}$-thick coronal sections. Series were stored at $-20^{\circ} \mathrm{C}$ in an anti-freeze solution until used for immunostaining.

Immunofluorescence. Sections were rinsed in PBS for $3 \times 10$ min and blocked in 4.5\% normal goat serum (NGS) diluted in PBS with 0.2\% Triton X-100 (PBST) for 1h at room temperature (RT). Sections were incubated overnight at $4^{\circ} \mathrm{C}$ with anti-GFAP-Cy3 antibody $(1: 1,000$, Sigma, \#C9205, Saint Louis, MO) diluted in 3\% NGS/PBST. Sections were rinsed $3 \times 10$ min in PBS and incubated with secondary Alexa Fluor-conjugated antibodies (1:1,000, Invitrogen, Carlsbad, CA) in 3\% NGS/PBST for $1 \mathrm{~h}$ at RT. After 3 washes in PBS, sections were incubated overnight at $4^{\circ} \mathrm{C}$ with an anti-GFP biotinylated antibody (1:500, Vector Laboratories, \#BA-0702, Burlingame, CA) in 3\% NGS/PBST. After 3 rinses in PBS, sections were incubated for $1 \mathrm{~h}$ at RT with Streptavidine-FITC $(1: 1,000$, ThermoFisher Scientific, Waltham, MA) in 3\% NGS/PBST and rinsed 3 times with PBS before being mounted on SuperFrost ${ }^{\circledR}$ Plus slides (ThermoFisher Scientific) and coverslipped with Fluorsave ${ }^{\mathrm{TM}}$ medium (Calbiochem, Darmstadt, Germany). For Iba1 (1:500, Wako, \#191974, Neuss, Germany) and Vimentin (1:750, Abcam, \#Ab24525, Cambridge) staining, a standard protocol was performed as described above but endogenous GFP was not amplified and sections were incubated with DAPI (1:2,000, Invitrogen, \#D21490) before being coverslipped with Vectashield ${ }^{\mathrm{TM}}$ medium (Vector Laboratories, \#H1400). For methoxy-XO4 (MXO4, Tocris, Minneapolis, MN) staining, sections were incubated with $33 \mu \mathrm{g} / \mathrm{ml}$ MXO4 in $0.1 \mathrm{M}$ PBS for $30 \mathrm{~min}$ at RT under mild agitation. After 3 rinses with PBS, a standard protocol for immunofluorescence was performed as described above. For A $\beta$ staining with the 4G8 antibody (1:500, Covance, \#SIG39240, Princeton, NJ), sections were treated with $70 \%$ formic acid for 2 min before incubation with primary biotinylated 4G8 antibody for $48 \mathrm{~h}$ at $4{ }^{\circ} \mathrm{C}$. After 3 rinses with PBS, sections were incubated for $1 \mathrm{~h}$ at RT with Streptavidine-Cy3 (1:1,000, Sigma) in 3.5\% NGS/PBST. Sections were then mounted as previously described.

Immunohistochemistry. Sections were rinsed in PBS for $3 \times 10$ min and incubated with $\mathrm{H}_{2} \mathrm{O}_{2}(1: 100$, Sigma) for $20 \mathrm{~min}$ at RT to inhibit peroxidases. For HT7 antibody, sections were pre-treated with 10 
$\mathrm{mM}$ citrate buffer for $20 \mathrm{~min}$ at $90^{\circ} \mathrm{C}$ before $\mathrm{H}_{2} \mathrm{O}_{2}$ treatment. Sections were then blocked in $4.5 \%$ NGS/PBST for $1 \mathrm{~h}$ at RT and incubated overnight at RT or $48 \mathrm{~h}$ at $4{ }^{\circ} \mathrm{C}$ in $3 \%$ NGS/PBST with the following primary antibodies: anti-A $\beta 42$ (1:500, Rabbit, Life Technologies, \#44-344, Carlsbad, CA), AT8 (1:400, Mouse, ThermoFisher Scientific, \#MN1020B), HT7 (1:1,000, Mouse, Innogenetics, \#90204, Zwijnaarde, Belgium), anti-phospho-Ser422-Tau (1:400, Rabbit, Abcam, \#Ab79415, Cambridge, UK). After $3 \times 10$ min rinses in PBS, sections were incubated for $1 \mathrm{~h}$ at RT with biotinylated secondary antibodies (1:4,000, except at 1:1,000 for anti-phospho-Ser422-Tau, Vector Laboratories) in 3\% NGS/PBST. They were washed $3 \times 10$ min and incubated for $1 \mathrm{~h}$ at RT with avidin/biotin complexes (1:250 in PBST, Vector Laboratories) and after 3 washes with PBS, incubated with VIP (Vector Laboratories). Sections were mounted on SuperFrost Plus slides and dried overnight at RT. Sections were dehydrated in acetone and xylene and coverslipped with Eukitt mounting medium (Chem-Lab, Zedelmgem, Belgium).

\section{Immunostaining quantification.}

GFAP staining was quantified on 10x-tiled images of serial brain sections ( $240 \mu \mathrm{m}$ between each sections), acquired with an epifluorescence microscope (BM6000B, Leica, Nussloch, Germany). Virally transduced $\mathrm{GFP}^{+}$regions were manually segmented on each section and the GFAP mean grey value was measured in this $\mathrm{GFP}^{+}$region with Image J. Vimentin was quantified on 10x-tiled images taken from another series of brain sections, acquired with the epifluorescence microscope. An intensity threshold was applied and the area of immunopositive pixels within the manually-segmented $\mathrm{GFP}^{+}$region were automatically extracted on each section. The Vimentin ${ }^{+}$volume was calculated by the Cavalieri method (West and Gundersen, 1990) (Volume $=\mathrm{t} \times \Sigma$ area; where $\mathrm{t}$ is the distance between two 2 sampled sections, here $240 \mu \mathrm{m}$ ). It was then normalized to the total $\mathrm{GFP}^{+}$volume, calculated by the same method. Representative fluorescent images in Fig. 1 and supplemental Fig. 2 were acquired with a 40x objective on a confocal microscope (TCS SP8, Leica).

The number of Iba1 positive cells around each plaque was quantified by scanning the whole slice on the $\mathrm{z}$ axis, under the 40x objective of the confocal microscope. An image was acquired at the center of the plaque to measure its cross-sectional area. In each mouse brain, 6-26 plaques within the GFP ${ }^{+}$ 
hippocampal region were sampled. They were taken from at least 3 fields of view from different sections.

Serial sections stained for HT7, Phospho-Ser422-Tau or AT8 were scanned under bright light, with an Axio scanZ.1 (Zeiss, Oberkochen, Germany) to cover the entire anteroposterior axis of the hippocampus. The immunopositive area within the manually-segmented hippocampus was automatically detected on each section, with an intensity threshold applied with Image J. The immunopositive volume was calculated by the Cavalieri method, normalized to the total volume of the hippocampus, calculated by the same method and expressed as a percentage. Hippocampal volume was not different between groups (data not shown).

The number and surface of all MXO4-labelled plaques were quantified on 10x-tiled fluorescent images of a series of slices covering the anteroposterior axis of the hippocampus, acquired on the Leica epifluorescence microscope. A $\beta 42$ plaques were quantified on immunostained sections scanned under bright field on the Axio scan. Automatic detection of objects according to intensity and size thresholds was performed on these images, after manual segmentation of the hippocampus on each section, with Image J. To quantify 4G8 immunoreactivity, the subiculum and stratum pyramidale of the CA1 region were manually segmented on 10x-tiled fluorescent images of a series of slices covering the anteroposterior axis of the hippocampus and the mean grey value was measured with Image J. Investigators were partially blinded to the group when analyzing immunostained sections, as the group can be deduced based on the presence of amyloid or Tau staining or GFP levels for example.

\section{Protein extraction.}

Hippocampal samples were homogenized by sonication (Labortechnik, Wasserburg, Germany, 6 strokes with cycle $=0.5,30 \%$ amplitude) in lysis buffer $[50 \mathrm{mM}$ Tris- $\mathrm{HCl} \mathrm{pH}=7.4,150 \mathrm{mM} \mathrm{NaCl}$, 1\% Triton X-100 with 1:100 phosphatase inhibitors (Sigma, cocktail 2) and 1x protease inhibitors (Roche, Basel, Switzerland)] and centrifuged at 20,000 g for $20 \mathrm{~min}$ at $4^{\circ} \mathrm{C}$. The supernatant contains Triton X-100-soluble proteins and was used for western blotting and MSD ${ }^{\circledR}$ ELISA tests. Protein concentration was measured by the BCA test (Pierce, Waltham, MA). 


\section{Western blot.}

Samples were diluted in loading buffer with DTT (NuPAGE® LDS sample buffer and sample reducing agent, Invitrogen). Ten $\mu \mathrm{g}$ of proteins were loaded on a NuPAGETM 4-12\% Bis-Tris Midi Gel (Life Technologies) and exposed to $200 \mathrm{~V}$ for 45 min in NuPAGETM Running Buffer (Invitrogen). Proteins were transferred on a nitrocellulose membrane with an iBlot Gel transfer device (Invitrogen). After 3 x 10 min rinses in Tris buffer saline and 0.1\% Tween 20 (TBST), membranes were blocked in $5 \%$ milk in TBST for $1 \mathrm{~h}$ at RT and incubated for $3 \mathrm{~h}$ at RT, or overnight at $4{ }^{\circ} \mathrm{C}$ with the following primary antibodies: 6E10 (1:500, Mouse, Covance, \#SIG-39320-20), anti-ApoE (1:1,000, Rabbit, Abcam, \#Ab20874), anti-BACE1 (1:1,000, Rabbit, Cell signaling, \#5606P, Danvers, MA), anti-GFAP (1:5,000, Rabbit, Dako, \#Z0334, Troy, MI), anti-IDE (1:400, Rabbit, Abcam, \#Ab32216), anti phospho-Ser404-Tau (1:1000, Rabbit, Sigma, \#T7444) and anti-Tubulin $\alpha$ (1:1,000, Mouse, Sigma, \#T5168). After $3 \times 10$ min washes in TBST, membranes were incubated for $1 \mathrm{~h}$ at RT with HRPconjugated secondary antibodies (1:5,000, Vector laboratories) diluted in TBST with 5\% milk. Membranes were incubated with the Clarity Western ECL substrate (Bio-Rad, Hercules, CA) and the signal was detected with a Fusion FX7 camera (ThermoFisher Scientific). Band intensity was quantified with ImageJ and normalized to Tubulin $\alpha$. Each antibody was used on at least 2 different membranes. Representative images and quantification are shown.

\section{MSD ${ }^{\circledR}$ ELISA tests.}

Triton $\mathrm{x}-100$ soluble proteins were diluted in the diluent provided for the V-PLEX A $\beta$ peptide panel kit $\left(\mathrm{A} \beta 38, \mathrm{~A} \beta 40, \mathrm{~A} \beta 42,6 \mathrm{E} 10\right.$ antibody, $\mathrm{MSD}{ }^{\circledR}$, Rockville, MD). For the phospho-Thr231-Tau/Total Tau kit $\left(\mathrm{MSD}{ }^{\circledR}\right)$, samples were diluted in $20 \mathrm{mM}$ Tris $\mathrm{HCl} \mathrm{pH}=7.4,150 \mathrm{mM} \mathrm{NaCl}, 1 \mathrm{mM}$ EDTA, 1 mM EGTA, 1\% Triton X-100, with phosphatase and protease inhibitors. For both assays, samples were loaded in triplicate and the manufacturer's protocol was followed. Peptide levels were quantified with a standard curve, using the Discovery Workbench4.0, MSD ${ }^{\circledR}$ software, and normalized to the protein content in each well. 


\section{Behavioral analysis.}

WT-GFP, 3xTg-GFP and 3xTg-SOCS3 mice were tested at the age of 8 and 14-15 months on the elevated plus maze to evaluate anxiety and after one month on the Y maze to assess working spatial memory. All tests were performed between 8 am and noon. Mice were handled daily for 2 min for $5 \mathrm{~d}$ before the first training session. Both mazes were cleaned with $10 \%$ ethanol and carefully dried between each phase and each mouse to remove odors. Investigators were blinded to the group when performing experiments.

The elevated plus maze test was formed by four connected arms placed $50 \mathrm{~cm}$ above the ground, illuminated at 100 lux in the center (see Fig. 6b). Two arms made of black Plexiglas were closed (nonstressful arms) while the two others were open (stressful arms). Each mouse was placed in the center of the maze and video-tracked for 5 min with the EthoVision XT11.5 software (Noldus IT, The Netherlands). The following parameters were measured: distance traveled and time spent in each arm, frequency of entry into each arm, duration and number of head dipping (counted manually during the experiment). Mice performing fewer than 4 entries into different arms were excluded from the analysis.

The Y maze test was composed of three arms in black Plexiglas, illuminated at 110 lux in the center. Each mouse was placed at the extremity of the "start arm" and left for a training session of 15 min, with one of the two other arms closed with a black Plexiglas door (the closed arm was randomly chosen for each mouse). Then, the mouse was brought back to its cage for $5 \mathrm{~min}$, the maze was cleaned, the closed arm was opened and the mouse placed at the extremity of the start arm for a 5 min test session, under videotracking (see Fig. 6a). The distance traveled and time spent in each arm (start, familiar, and novel arms) were measured and analyzed with EthoVision. Given their natural curiosity, mice are expected to spend more time exploring the new arm. Mice that stayed in the start arm during training or made less than 4 total entries in the 3 arms during testing were excluded.

\section{Statistical analysis.}

Results are expressed as mean \pm SEM. Statistical analysis were performed with Statistica software (StatSoft, Tulsa, OK). Student $t$ test and one-way ANOVA and Tukey's post hoc test were used to 
compare two and three groups, respectively. Two-way (group, arm) ANOVA followed by Tuckey's post-hoc test was used for Y maze analysis. For each analysis, normality of variables or residues and homoscedasticity were assessed. If any or both conditions of application were not fulfilled, nonparametric tests were used. Two groups were compared by the Mann-Whitney test and 3 independent groups were compared by a Kruskal-Wallis test followed by a Mann-Whitney test. The significance level was set at $p<0.05$. The number of analyzed mice per group is indicated in each bar histogram.

\section{RESULTS}

\subsection{The JAK2-STAT3 pathway efficiently controls reactive features of hippocampal astrocytes in $3 x T g$ mice.}

We previously showed that adeno-associated viral (AAV) gene transfer of SOCS3, an inhibitor of the JAK-STAT3 pathway, in hippocampal astrocytes, prevents their acquisition of reactive molecular features, including in 9 and 12-month-old 3xTg mice (Ben Haim et al., 2015b; Ceyzeriat et al., 2018). We studied whether SOCS3 was still able to block reactive astrocytes at a later stage, when both Tau and amyloid pathologies are present in 16-month-old 3xTg mice.

Six-month-old female 3xTg mice were injected with an AAV2/9 vector encoding Socs 3 under the gfaABC $C_{1} \mathrm{D}$ promoter ( $3 \mathrm{xTg}$-SOCS3 mice). This viral vector allows the selective transduction of astrocytes, in the dorsal hippocampus (Fig. 1a, Supplemental Fig. 2), covering 25\% of the whole hippocampus. WT and 3xTg mice were injected with an AAV encoding GFP as control (WT-GFP and 3xTg-GFP mice respectively, see Supplemental Fig. 1). Brain sections from 16-month-old 3xTg-GFP mice displayed 3-fold higher GFAP levels than WT-GFP mice (Fig. 1b, c). In 3xTg-GFP mice, hippocampal astrocytes appear hypertrophic, a typical hallmark of reactive astrocytes. AAV-gene transfer of SOCS3 in astrocytes reduced GFAP levels and normalized astrocyte morphology (Fig. 1b, c).

Mirror experiments were performed on another mouse cohort to further increase the activation of astrocytes by stimulation of the JAK2-STAT3 pathway. Three-month-old female 3xTg mice were injected in the hippocampus with an AAV encoding a constitutively active form of JAK2 (JAK2ca), the upstream kinase of the JAK2-STAT3 pathway, and were studied at 16 months (3xTg-JAK2ca 
mice). Control groups included WT-GFP and 3xTg-GFP mice injected and analyzed at the same age (Supplemental Fig. 1). 3xTg-JAK2ca mice displayed enhanced astrocyte reaction, visible as a twofold increase in GFAP immunoreactivity and exacerbated enlargement and tortuosity of astrocyte processes, compared with 3xTg-GFP mice (Fig. 1b, c). Modulation of GFAP levels by SOCS3 and JAK2ca was also observed by western blotting on hippocampal samples taken from WT-GFP, 3xTgGFP, 3xTg-SOCS3 and 3xTg-JAK2ca mice (Fig. 1e). The immunoreactivity for vimentin, another intermediate filament characteristic of reactive astrocytes, was also reduced by more than $50 \%$ by SOCS3 in the hippocampus of 3xTg mice, although it did not reach significance (Fig. 1b, d).

Conversely, vimentin immunoreactivity was increased more than 3 -fold by JAK2ca (Fig. 1b, d). The number of microglia cells in direct contact with amyloid plaques was not impacted by either SOCS3 or JAK2ca in 3xTg-AD mice (Fig. 2a, b), suggesting that our strategy modulates reactive astrocytes without significantly impacting microglial cells around plaques, as reported in another mouse model of $\mathrm{AD}$ (Ceyzeriat et al., 2018).

These results show that manipulation of the JAK2-STAT3 pathway in hippocampal astrocytes is an efficient strategy to modulate the reactive state of astrocytes in 3xTg mice. We next evaluated how manipulation of reactive astrocytes by SOCS3 and JAK2ca impacted Tau hyper-phosphorylation and amyloid deposition, two cardinal features of AD.

\subsection{Modulation of reactive astrocytes does not influence Tau phosphorylation in $3 x T g$ mice.}

At the age of 16 months, 3xTg mice display both Tau and amyloid pathologies. Tau can be phosphorylated on multiple epitopes. Three main approaches were used to characterize the effects of reactive astrocytes on Tau phosphorylation: i) immunostainings with antibodies against phosphoSer422-Tau, phospho-Ser202/Thr205-Tau (AT8 antibody) and total human Tau (HT7 antibody) as a control, ii) ELISA MSD ${ }^{\circledR}$ assay with antibodies against phospho-Thr231-Tau (AT180 antibody) and total Tau and iii) western blotting against phospho-Ser404-Tau.

Positive staining for HT7, phospho-Ser422-Tau and AT8 was observed in 3xTg mice but not in WT mice (Fig. 3a). HT7 immunoreactivity was not changed by SOCS3 or JAK2ca in the 
hippocampus of 3xTg mice (Fig. 3a, b), suggesting that the expression of the human Tau transgene is not impacted by our experimental manipulations. Immunostaining with phospho-Ser422-Tau and AT8 antibodies revealed dystrophic neurons and numerous ghost tangles in the hippocampus (Fig. 3a). Immunoreactivity for these two antibodies was similar between the 3xTg-GFP, 3xTg-SOCS3 and 3xTg-JAK2ca groups (Fig. 3b), suggesting that modulation of reactive astrocytes through the JAK2STAT3 pathway does not impact the level of phosphorylation of these epitopes.

We next measured hippocampal concentrations of phospho-Thr231-Tau and total human Tau by MSD ${ }^{\circledR}$ assays on protein homogenates prepared from the hippocampus of 3xTg-GFP, 3xTg-SOCS3 or 3xTg-JAK2ca. Levels of phospho-Thr231-Tau normalized by total Tau and total Tau were not impacted by SOCS3 or JAK2ca in 16-month-old 3xTg mice (Fig. 3c). To assess whether reactive astrocytes impact Tau phosphorylation at a later stage of the disease, we performed the same measurement in 26-month-old female 3xTg mice that were injected 2 months before, with AAV-GFP or AAV-SOCS3. Tau phosphorylation on Thr231 was also unaffected by SOCS3 in 26-month-old 3xTg mice (Fig. 3d).

Lastly, we analyzed phospho-Ser404-Tau levels by western blotting on hippocampal protein samples from 16-month-old 3xTg-GFP, 3xTg-SOCS3 and 3xTg-JAK2ca. We found no difference between the groups (Supplemental Fig. 3).

Our results show that JAK2-STAT3 pathway modulation of reactive astrocytes in 3xTg mice does not influence Tau phosphorylation on several epitopes, at advanced and terminal stages of the disease.

\subsection{Modulation of reactive astrocytes does not impact amyloid deposition in 3xTg mice.}

Amyloid pathology was assessed by four approaches: i) fluorescent staining with the Congo-red derivative Methoxy-XO4 (MXO4) that binds dense-core amyloid plaques of different size, ii) immunostaining with the A $\beta 42$ antibody that labels small dense-core plaques (Serrano-Pozo et al., 2011) iii) immunofluorescent staining with the $4 \mathrm{G} 8$ antibody that labels diffuse plaques, as well as intracellular A $\beta$, which is prominent in 3xTg mice (Oddo et al., 2003), and finally iv) MSD ${ }^{\circledR}$ measurement of Triton X100-soluble A $\beta$ levels in hippocampal homogenates. 
In 16-month-old 3xTg mice, numerous $\mathrm{MXO}^{+}$plaques were found in the subiculum and CA1 region of the hippocampus (Fig. 4a). Modulation of reactive astrocytes by SOCS3 or JAK2ca had no detectable impact on hippocampal amyloid plaques stained by MXO4 (Fig. 4a). Detection of amyloid plaques by immunostaining with the $\mathrm{A} \beta 42$ antibody also showed no difference in their number between the three groups (Supplemental Fig. 4). The average individual size of $\mathrm{MX04}^{+}$or $\mathrm{Ab}_{2} 2^{+}$ plaques was identical between groups (Fig. 4b, c and Supplemental Fig. 4, respectively). Similarly, immunoreactivity in the subiculum and pyramidal layer of the CA1 region for intracellular A $\beta$ labelled by the 4G8 antibody was identical between groups (Supplemental Fig. 4).

We next measured Triton X-100 soluble $\mathrm{A} \beta$ levels by MSD® assays on protein homogenates prepared from the hippocampus of 3xTg-GFP, 3xTg-SOCS3 or 3xTg-JAK2ca mice. At the age of 16 months, only A $\beta 42$ was reliably detected and quantified (A $\beta 40$ was undetectable in 2 to 4 mice in each group, corresponding to $30 \%$ of the two cohorts). Hippocampal A $\beta 42$ concentrations were not significantly modified by SOCS3 or JAK2ca (Fig. 5a). Accordingly, several proteins involved in APP metabolism were found at similar levels in 16-month-old in 3xTg-GFP, 3xTg-SOCS3 and 3xTgJAK2ca mice [APP; BACE1, the $\beta$-secretase that cleaves APP through the amyloïdogenic pathway; insulin degrading enzyme (IDE) that degrades A $\beta$; and apolipoprotein E (ApoE) a protein secreted by astrocytes, which binds and clears cholesterol and A $\beta$ (Supplemental Fig. 5)].

To study whether reactive astrocytes impact $A \beta$ levels at an earlier or later stage of the disease, we performed the same measurement of A $\beta$ levels in 9-month-old and 26-month-old female 3xTg-GFP and 3xTg-SOCS3 mice. Again, A $\beta 42$ concentrations were identical between groups at both ages and A 338 and A 340 only reached detectable levels in 26-month-old 3xTg mice (Fig. 5b, c). The $\mathrm{A} \beta 42 / \mathrm{A} \beta 40$ ratio was not impacted by SOCS3 but concentrations of $\mathrm{A} \beta 38$, the least toxic form ( $\mathrm{Li}$ et al., 2018), were significantly decreased by SOCS3 (Fig. 5c).

Overall, our analysis demonstrates that JAK2-STAT3-dependent reactive astrocytes do not overtly contribute to amyloid metabolism and deposition in 3xTg mice, at three different disease stages. 


\subsection{Inhibition of reactive astrocytes improves anxiety but not memory in $3 x \mathrm{Tg}$ mice.}

We previously showed that hippocampal reactive astrocytes contribute to deficits in synaptic transmission and plasticity in 9-month-old 3xTg mice (Ceyzeriat et al., 2018). Here, we analyzed whether synaptic restoration by SOCS3 translates into cognitive improvement in 3xTg mice.

We tested whether SOCS3-mediated inhibition of reactive astrocytes improved spatial memory deficits on the Y maze (Carroll et al., 2007) and anxiety on the elevated plus maze (Blanchard et al., 2010). WT-GFP, 3xTg-GFP mice and 3xTg-SOCS3 were first tested at 8 months. Each mouse was placed on the Y maze to explore two arms (the start and familial arms, one being closed) for 15 min of training (Fig. 6a). After $5 \mathrm{~min}$ in their home cage, the mouse was placed again in the start arm of the maze with the three arms opened. In average, WT-GFP, 3xTg-GFP and 3xTg-SOCS3 mice traveled the same total distance during the test session, showing equivalent exploration (Fig. 6a). However, they explored the three arms differently ( $p<0.001$ for Arm effect, two-way ANOVA, Fig. 6a). WTGFP mice displayed the expected preference for the novel arm during the test session. On the contrary, 3xTg-GFP and 3xTg-SOCS3 mice did not discriminate the new arm compared to the two other arms, revealing an alteration in working memory (Fig. 6a). These mice were also tested on the elevated plus maze. The time spent in the open arms of the maze was used as a surrogate for the level of anxiety. All three groups explored similarly the open arms, suggesting that 3xTg mice did not display higher anxiety at this age (Fig. 6b).

These mice were tested again at the age of 14 months. At this later stage, WT-GFP mice displayed an even stronger preference for the new arm on the test phase (Fig. 6a, $p<0.001$ for Group $\mathrm{x}$ Arm effect, two-way ANOVA) whereas 3xTg-GFP mice explored significantly more the start arm than the novel and familiar arms (Fig. 6a). This lack of preference for the novel arm confirms the alteration in working memory, while the preference for the start arm suggests increased anxiety to explore more distant arms. SOCS3 did not improve working spatial memory in 3xTg mice, but it reduced their anxiety, as they did not remain in the start arm and explored the three arms similarly (Fig. 6a).

On the elevated plus maze, 3xTg-GFP mice tended to spend less time and to perform less head dipping in open arms, an indication of higher anxiety. SOCS3 reduced anxiety in 3xTg mice, by significantly increasing the time spent in the stressful open arms (Fig. 6b). 
Our data show that inhibition of reactive astrocytes by SOCS3 does not alleviate memory deficits in $3 \times \mathrm{Tg}$ mice but reduces anxiety levels.

\section{DISCUSSION}

Our multi-level preclinical analysis shows that modulation of hippocampal reactive astrocytes through the JAK2-STAT3 pathway does not significantly impact Tau or amyloid pathologies, at different stages in 3xTg-AD mice. However, SOCS3-mediated inhibition of reactive astrocytes reduced their anxiety, without restoring short-term spatial memory.

\subsection{The JAK2-STAT3 pathway and reactive astrocytes.}

This study further demonstrates the central role played by the JAK2-STAT3 pathway in the control of reactive astrocytes as previously reported in other mouse models of neurodegenerative diseases (Ben Haim et al., 2015b; Ceyzeriat et al., 2018; Reichenbach et al., 2019) and other diseases like ischemia, spinal cord injury or trauma (see, Ceyzériat et al., 2016 for review). Strikingly, we found that expression of SOCS3 or JAK2ca in astrocytes was able to respectively inhibit or exacerbate GFAP expression and morphological changes in hippocampal reactive astrocytes of $3 \times \mathrm{Tg}$ mice. These effects are extremely stable (observed after $>10$ months post-injection) and allow analysis of reactive astrocytes at different disease stages. Previously, we have shown that, besides these two hallmarks, SOCS3 was also able to normalize the transcriptome of reactive astrocytes in neurodegenerative disease models (Ben Haim et al., 2015b; Ceyzeriat et al., 2018). It would be interesting to explore the molecular profile of astrocytes in 3xTg-GFP and 3xTg-SOCS3 mice as well, to assess whether the same genes are dysregulated in these two AD models, and whether they are normalized by SOCS3. Similar reduction in reactive gene levels was found with the conditional knock-out of STAT3 in astrocytes in APP/PS1dE9 mice (Reichenbach et al., 2019) and mice with spinal cord injury (Anderson et al., 2016). Thus, activation of the JAK2-STAT3 pathway is necessary for both the induction and persistence of several molecular and morphological features of reactive astrocytes, identifying this cascade as a master regulator of astrocyte reaction in disease. 


\subsection{Reactive astrocytes do not influence molecular hallmarks of AD in 3xTg mice.}

We show that JAK2-STAT3-dependent reactive astrocytes do not significantly impact Tau and amyloid pathologies. This was observed at three different stages of the disease (9,16 and 24 months), ruling out stage-dependent effects. We found that $30 \%$ of $3 \times \mathrm{Tg}$ mice did not display detectable A $\beta 40$ levels at 16 months. In the brain of $3 x T g$ mice, A $\beta 40$ levels are either reported to be higher than those of A $\beta 42$ (Hirata-Fukae et al., 2008), equivalent (ㅁdo et al., 2003) or lower (Goni et al., 2013). A 340 levels are probably dependent on the exact brain region analyzed and the mouse age, as well as the extraction protocol and detection method. A $\beta 40$ was readily detected in 26-month-old mice but its levels remained lower than those of $A \beta 42$. We only found a significant reduction in $A \beta 38$ levels in the hippocampus of end-stage 3xTg-SOCS3 mice. A $\beta 40$ and A $\beta 42$ tended to be reduced in this group as well, but it did not reach significance. It is possible that a higher number of mice per group would lead to significant differences between groups but nonetheless, the effects would remain small (20-30\% range). $A \beta 38$ was shown to be less synaptotoxic than $A \beta 40$ or $A \beta 42$ (Li et al., 2018), suggesting that such an end-stage and marginal reduction in A $\beta 38$ levels may not significantly alleviate neuronal dysfunctions. Therefore, JAK2-STAT3-dependent reactive astrocytes have only a minor role in amyloid processing in this model.

The lack of SOCS3 effects on amyloid deposition is in contrast with our previous study reporting a significantly reduced number of $\mathrm{MXO}^{+}$amyloid plaques in APP/PS1dE9 mice with the same AAVSOCS3 (Ceyzeriat et al., 2018). This AAV also infected 25\% of hippocampal astrocytes in these mice, showing that even a partial inhibition of reactive astrocytes in the hippocampus is able to impact amyloid deposition. The present result is also in opposition with two studies reporting lower amyloid deposition after inhibition of reactive astrocytes through the calcineurin-NFAT pathway (Furman et al., 2012; Sompol et al., 2017) or after STAT3 conditional knockout in astrocytes of APP/PS1dE9 mice (Reichenbach et al., 2019). However these two strategies to interfere with reactive astrocytes could impact a different set of astrocyte molecular and functional features than SOCS3 expression, leading to different effects on amyloid deposition. The fact that the same experimental manipulation of reactive astrocytes (through the JAK2-STAT3 pathway) in two AD mouse models have different 
effects on the same disease outcome (amyloid plaques), assessed with the same method (MXO4) underlines that astrocyte reaction is highly context-dependent. How can the discrepancies between these two AD models be explained? In 16-month-old 3xTg mice, both amyloid and Tau pathologies are present, whereas only amyloid pathology develops in APP/PS1dE9 mice. This important difference (in addition to other differences such as sex, genetic background, and genetic constructs) could change the mechanisms of amyloid production, accumulation, aggregation and clearance. In addition, mutant Tau itself impacts astrocyte physiology (Dabir et al., 2006; Leyns and Holtzman, 2017) and could directly change their ability to clear amyloid.

Most importantly, accumulating evidence shows that astrocytes and their reactive counterparts are heterogeneous. Reactive astrocytes may adopt different molecular and functional states depending on the pathological context, and they could subsequently influence disease processes differentially (Anderson et al., 2014; Escartin et al., 2019). Therefore, even if the causes for SOCS3 differential impact on amyloid deposition in 3xTg and APP/PS1 dE9 mice were not identified, our studies illustrate that reactive astrocytes have complex and subtle effects in vivo, and call for caution in generalizing their roles in disease. In particular, this study was performed on female mice, and the same experiments should be reproduced in males.

\subsection{Inhibition of reactive astrocytes does not restore spatial memory but reduces anxiety in}

\section{$3 x T g$ mice.}

We previously reported that SOCS3 expression in astrocytes fully restored early synaptic and LTP alterations in 9-month-old 3xTg mice (Ceyzeriat et al., 2018). Here, we explored whether this normalization of synaptic activity translated into improved short-term memory and reduced anxiety in 3xTg mice. We found that 8-month-old 3xTg mice displayed working memory alterations on the $\mathrm{Y}$ maze and normal levels of anxiety on the elevated plus maze. SOCS3 did not influence this behavioral deficit at this early stage. At 14 months, 3xTg mice displayed altered spatial memory and increased anxiety. Unexpectedly, blocking reactive astrocytes with SOCS3 reduced anxiety but did not improve working memory. 
The absence of SOCS3 effect on working memory could be explained by the fact that this type of memory involves other brain structures besides the targeted hippocampus. For example, it is now admitted that the prefrontal cortex is involved in spatial information processing (Funahashi, 2017). Accordingly, we found that SOCS3 was able to improve spatial learning but not memory retrieval on the Morris water maze in APP/PS1dE9 mice (Ceyzeriat et al., 2018), further stressing the role of complex brain circuits for memory tasks. A strategy targeting the JAK-STAT3 pathway in a larger part of the brain through multiple injections with i.v. delivery of blood brain barrier permeant vectors encoding SOCS3 or pharmacological approaches would help uncover the effects of reactive astrocytes on memory impairment in $\mathrm{AD}$ mice.

Conversely, reduced anxiety after AAV-SOCS3 injection in the dorsal hippocampus of 3xTgSOCS3 mice was unexpected, because anxiety-related behaviours are thought to be controlled by the ventral hippocampus and amygdala (Bannerman et al., 2004; Calhoon and Tye, 2015). However, recent data describe extensive connections within subfields of the hippocampus and between the hippocampus and other limbic areas (Bienkowski et al., 2018). Therefore, it is possible that targeting only a fraction of the hippocampus can impact larger brain circuits that regulate anxiety. Another nonexclusive possibility is that that gap junction-based astrocyte networks could propagate SOCS3 effects and influence distant hippocampal neurons.

Anxiety-related behaviors were evaluated on the elevated plus maze, as the mouse propensity to explore anxiogenic open arms. Additional tests like the open field would be useful to better delineate anti-anxiogenic effects of SOCS3 in 3xTg mice. Interestingly, we observed that 3xTg mice remained in the start arm of the Y maze, an indication of increased anxiety and this was corrected by SOCS3 as well, further demonstrating its positive effect on anxiety.

When do reactive astrocytes start to play a role in $\mathrm{AD}$ pathogenesis? Reactive glial cells (astrocytes and microglia) appear in response to an abnormal environment [e.g. dysfunctional neurons, presence of toxic oligomerized proteins (see, Ben Haim et al., 2015a, for review)]. But, positron emission tomography studies on prodromal $\mathrm{AD}$ patients showed that reactive glial cells may be present at early disease stages, before overt signs of amyloid deposition (Hamelin et al., 2016; Heneka et al., 2005). In accordance, our results indicate that reactive astrocytes contribute to early synaptic deficits, before 
amyloid deposition (Ceyzeriat et al., 2018) and their inhibition may improve some behavioral outcomes without reducing $A \beta$ and Tau pathologies.

\section{CONCLUSIONS}

Overall, the JAK2-STAT3 pathway appears as a core signaling cascade for reactive astrocytes. By modulating this pathway in different models of neurodegenerative diseases, we show that reactive astrocytes are multifaceted cells, with subtle functional effects, depending on the specific disease context. Our vector-based strategy is versatile and efficient to dissect out the roles of JAK2-STAT3dependent reactive astrocytes in different pathological conditions. A better understanding of the molecular changes occurring in reactive astrocytes and their impact on neurons is required to devise efficient therapeutic strategies targeting astrocytes. 


\section{FIGURE LEGENDS}

Figure 1. Long-term modulation of reactive astrocytes by targeting the JAK2-STAT3 pathway in $3 x T g$ mice.

a, Low magnification image of serial brain sections of a mouse injected with an AAV-GFP in CA1. GFP (green) is expressed throughout the dorsal hippocampus. b, Confocal images of GFP ${ }^{+}$astrocytes (green) stained for GFAP (magenta) or vimentin (cyan) in 16-month-old WT and 3xTg mice injected in the CA1 region with AAV-GFP, AAV-SOCS3 + AAV-GFP or AAV-JAK2ca + AAV-GFP (same viral titer). In 3xTg mice, astrocytes display the typical reactive features (GFAP overexpression and morphological changes). c-d, SOCS3 significantly reduces GFAP levels (c) and tends to reduce vimentin levels (d), while JAK2ca increases both GFAP and vimentin levels $* p<0.05, * * p<0.01$, *** $p<0.001$. Kruskall-Wallis and Mann-Whitney tests. e, Western blotting and quantification of GFAP levels (normalized to tubulin $\alpha$ ) in WT-GFP, 3xTg-GFP, 3xTg-SOCS3 and 3xTg-JAK2ca mice show the same pattern of GFAP modulation by SOCS3 and JAK2ca. One-way ANOVA and Tukey's post hoc test (Left graph: $\mathrm{F}(2,19)=4.724, * p<0.05$; right graph: $\mathrm{F}(2,18)=5.267, * p<0.05)$. Quantifications are expressed relatively to the WT group in each cohort, whose value was set to 1.

\section{Figure 2. Modulation of reactive astrocytes does not impact the number of microglial cells}

\section{around plaques in $3 x T g$ mice.}

a, Representative confocal image of $\mathrm{Iba}^{+}$microglia cells (magenta, arrowhead) around amyloid plaque (cyan, star) in 16-month-old 3xTg-GFP, 3xTg-SOCS3 and 3xTg-JAK2ca mice. Nuclei and amyloid plaques are labelled with DAPI (cyan). b, Quantification of the number of $\mathrm{Iba}^{+}{ }^{+}$cells per plaque shows no difference between groups. Student $t$ test (Upper graph: $\mathrm{t}(15)=-0.495, p=0,628$; lower graph: $\mathrm{t}(13)=-0.320, p=0.754)$.

Figure 3. Modulation of reactive astrocytes does not impact Tau pathology in 3xTg mice.

a, Representative brain sections of 16-month-old WT, 3xTg-GFP, 3xTg-SOCS3 and 3xTg-JAK2ca mice stained with antibodies against total human Tau (HT7), phospho-Ser422-Tau (P-Ser422-Tau) or 
phospho-Ser202/Thr205-Tau (AT8). High magnification images for each staining shows dystrophic neurons and ghost tangles in 3xTg mice. b, Quantification of the immunopositive volume in the whole hippocampus shows no difference between groups for these three markers. Student $t$ test (AT8 left graph: $\mathrm{t}(15)=-0.558, p=0.585$; AT8 right graph: $\mathrm{t}(12)=2.001, p=0.069)$ or Mann-Whitney test

(HT7, P-Ser422-Tau). c, MSD ${ }^{\circledR}$ measurement of phospho-Thr231-Tau (AT180 antibody) and total Tau in protein homogenates prepared from the hippocampus of 16-month-old 3xTg-GFP, 3xTgSOCS3 and 3xTg-JAK2ca mice. Total Tau and AT180/Tau levels are not impacted by SOCS3 or JAK2ca in 3xTg mice. Mann-Whitney test. d, The same measurement in 26-month-old 3xTg-GFP and 3xTg-SOCS3 mice shows that total Tau and AT180/Tau levels are similar in both groups. MannWhitney test.

Figure 4. Modulation of reactive astrocytes does not impact amyloid load in 16-month-old $3 x T g$ mice.

a, Representative images of amyloid plaques labelled with MXO4 (cyan) in 16-month-old 3xTg-GFP, 3xTg-SOCS3 and 3xTg-JAK2ca mice. b, Quantification of the total number of MXO4 ${ }^{+}$plaques in the whole hippocampus shows no difference between groups. Mann-Whitney test. c, The mean size of individual $\mathrm{MXO}^{+}$amyloid plaques is also similar between groups. Mann-Whitney test.

Figure 5. Modulation of reactive astrocytes does not impact soluble Aß levels in 3xTg mice. $\mathrm{MSD}{ }^{\circledR}$ measurement of $\mathrm{A} \beta 42$ concentration in Triton-X100 soluble protein homogenates prepared from the hippocampus of 16-month-old 3xTg-GFP, 3xTg-SOCS3 and 3xTg-JAK2 ca mice (a), or in 3xTg-GFP and 3xTg-SOCS3 mice at the age of 9 (b) and 26 (c) months. A 342 levels increase with age, but are not changed by SOCS3 or JAK2ca in 3xTg mice. c, A $\beta 40$ levels, A $\beta 42 / 40$ ratio are similar in 3xTg-GFP and 3xTg-SOCS3 groups, while A 338 levels are significantly reduced by SOCS3 in end-stage 26-month-old 3xTg mice. $* p<0.05$. Mann-Whitney test. 


\section{Figure 6. SOCS3-inbition of reactive astrocytes does not correct memory deficits but}

\section{reduces anxiety in $3 x T g$ mice at 14 months.}

a, The total distance traveled during the test phase of the Y maze is not different between the three groups, at both 8 and 14 months $(8$ months: $\mathrm{F}(2,19)=1.789, p=0.194 ; 14$ months: $\mathrm{F}(2,13)=0.156, p$ $=0.857)$. At 8 months, WT-GFP mice preferentially explore the novel arm $(\mathrm{N})$ over the start $(\mathrm{S})$ or the familiar arm (F). In contrast, 3xTg-GFP and 3xTg-SOCS3 show no preference for the novel arm, reflecting an altered working memory. Similarly, at 14-months, WT-GFP still explore preferentially the novel arm while 3xTg-GFP mice show no preference for the novel arm. They traveled significantly more distance in the start arm than WT-GFP mice, suggesting increased anxiety. Interestingly, 3xTgSOCS3 mice did not stay within the start arm and explored all arms similarly, suggesting that SOCS3 reduces mouse anxiety but does not correct short-term memory. Two-way (group, arm) ANOVA and Tuckey post-hoc test [8 months: Arm effect $\mathrm{F}(2,57)=17.444 p<0.001$, Group x Arm interaction $\mathrm{F}(4$, $57)=1.3734, p=0.254 .14$ months: Arm effect $\mathrm{F}(2,39)=6.724, p<0.01$, Group $\mathrm{x}$ Arm interaction $\mathrm{F}(4,39)=8.220$ ), $p<0.001] .{ }^{*} p<0.05, * * p<0.01, * * * p<0.001$ (between arms, within the same group), $\# p<0.05$ (between groups, for the same arm). b, At 8 months, the three mouse groups explored similarly the open arm on the elevated plus maze test. Kruskal-Wallis test. At 14 months, 3xTg-GFP mice tend to spend less time and perform less head dipping in open arms than WT-GFP mice. SOCS3 expression in hippocampal astrocytes significantly increases the time spent in open arms. Time in open arm: ANOVA and Tuckey post-hoc test, $\mathrm{F}(2,21)=3.702,{ }^{*} p<0.05$. Head dipping: Kruskal-Wallis test. 


\section{Supplemental figure 1. Experimental design.}

Four independent cohorts of female 3xTg mice and age- and gender-matched WT controls were injected and analyzed at the indicated ages.

\section{Supplemental figure 2. AAV infect astrocytes selectively in 3xTg mice.}

AAV2/9 encoding GFP under a gfaABC ${ }_{1} \mathrm{D}$ promoter were injected in the hippocampus of $3 \times \mathrm{xT}$-AD mice. All $\mathrm{GFP}^{+}$cells co-express the astrocytic marker GFAP (magenta) and display the typical ramified and bushy morphology of astrocytes. $\mathrm{GFP}^{+}$cells do not colocalize with microglial cells labelled with IBA1 (magenta) or with neurons in the CA1 pyramidal cell layer, visualized with DAPI staining (blue).

Supplemental figure 3. Modulation of reactive astrocytes does not impact phospho-Ser404Tau levels in $3 x T g$ mice.

Representative western blotting and quantification of phospho-Ser404-Tau (P-Ser404-Tau) in 3xTgGFP, 3xTg-SOCS3 and 3xTg-JAK2ca mice. P-Ser404-Tau levels (normalized by tubulin $\alpha$ ) are similar between groups. Mann-Whitney test. Quantifications are expressed relatively to the 3xTg-GFP group in each cohort, whose value was set to 1 .

\section{Supplemental figure 4. Modulation of reactive astrocytes does not impact amyloid}

\section{pathology in $3 x T g$ mice.}

a, Representative images of amyloid plaques labelled with A $\beta 42$ antibody in 16-month-old 3xTg-GFP, 3xTg-SOCS3 and 3xTg-JAK2ca mice. b, Quantification of A $\beta 42^{+}$plaques in the whole hippocampus. The number (b) and average individual size (c) of plaques are not impacted by SOCS3 or JAK2ca. N =9/group. Mann-Whitney test. d, Quantification of 4G8 immunoreactivity in the subiculum and stratum pyramidale of 16-month-old 3xTg-GFP, 3xTg-SOCS3 and 3xTg-JAK2ca mice. There is no difference in 4G8 signal between groups. Mann-Whitney test. Quantifications in $\mathbf{d}$ are expressed relatively to the $3 \times \mathrm{xTg}$-GFP group in each cohort, whose value was set to 1 . 


\section{Supplemental figure 5. Modulation of reactive astrocytes does not impact proteins involved}

in Aß metabolism or clearance in $3 x T g$ mice.

a, Representative western blotting and quantification of human APP (hAPP) and key enzymes (BACE1, IDE) or transporter (ApoE) involved in A $\beta$ production or clearance in 16-month-old 3xTgGFP, 3xTg-SOCS3 and 3xTg-JAK2ca. Protein levels are similar between groups. Mann-Whitney test. Quantifications are expressed relatively to the 3xTg-GFP group in each cohort, whose value was set to 1.

\section{Acknowledgements}

We are grateful to D. Cheramy and Dr. E. Diguet (Servier) for sharing their expertise and equipment for MSD® kits. We thank A. Panatier and S.H.R. Oliet (NeuroCentre Magendie, Bordeaux) for providing 4-month-old 3xTg mice of the $3^{\text {rd }}$ cohort, as well as D. Gonzales, S. Laumond, J. Tessaire and people at the animal facility of the NeuroCentre Magendie for mouse care and genotyping. We thank L. Vincent, K. Bastide, P. Woodling and J.M. Hélies for their help with mouse housing and transfer. We are very grateful to Dr. J. Martin for his careful reading of the manuscript.

\section{Funding sources}

This study was supported by CEA, CNRS and grants from the French National Research Agency (grants \# 2010-JCJC-1402-1, 2011-BSV4-021-03 and ANR-16-TERC-0016-01 to CE, and 2011INBS-0011 NeurATRIS to PH), from Fondation Vaincre Alzheimer (grant \# FR-15015 to CE) and Association France Alzheimer and Fondation de France (Prix Spécial 2012 to GB). CE received support from the Fédération pour la Recherche sur le Cerveau. OG, KC and LBH are recipients of a doctoral fellowship from the CEA. 


\section{$\underline{\text { REFERENCES }}$}

Abeti, R., Abramov, A.Y., Duchen, M.R., 2011. Beta-amyloid activates PARP causing astrocytic metabolic failure and neuronal death. Brain 134(Pt 6), 1658-1672.

Allaman, I., Gavillet, M., Belanger, M., Laroche, T., Viertl, D., Lashuel, H.A., Magistretti, P.J., 2010. Amyloid-beta aggregates cause alterations of astrocytic metabolic phenotype: impact on neuronal viability. J Neurosci 30(9), 3326-3338.

Anderson, M.A., Ao, Y., Sofroniew, M.V., 2014. Heterogeneity of reactive astrocytes. Neurosci Lett $565,23-29$.

Anderson, M.A., Burda, J.E., Ren, Y., Ao, Y., O'Shea, T.M., Kawaguchi, R., Coppola, G., Khakh, B.S., Deming, T.J., Sofroniew, M.V., 2016. Astrocyte scar formation aids central nervous system axon regeneration. Nature 532(7598), 195-200.

Arranz, A.M., De Strooper, B., 2019. The role of astroglia in Alzheimer's disease: pathophysiology and clinical implications. Lancet Neurol.

Arriagada, P.V., Growdon, J.H., Hedley-Whyte, E.T., Hyman, B.T., 1992. Neurofibrillary tangles but not senile plaques parallel duration and severity of Alzheimer's disease. Neurology 42(3 Pt 1), 631639.

Bannerman, D.M., Rawlins, J.N., McHugh, S.B., Deacon, R.M., Yee, B.K., Bast, T., Zhang, W.N., Pothuizen, H.H., Feldon, J., 2004. Regional dissociations within the hippocampus--memory and anxiety. Neuroscience and biobehavioral reviews 28(3), 273-283.

Ben Haim, L., Carrillo-de Sauvage, M.A., Ceyzeriat, K., Escartin, C., 2015a. Elusive roles for reactive astrocytes in neurodegenerative diseases. Frontiers in cellular neuroscience 9, 278.

Ben Haim, L., Ceyzeriat, K., Carrillo-de Sauvage, M.A., Aubry, F., Auregan, G., Guillermier, M., Ruiz, M., Petit, F., Houitte, D., Faivre, E., Vandesquille, M., Aron-Badin, R., Dhenain, M., Deglon, N., Hantraye, P., Brouillet, E., Bonvento, G., Escartin, C., 2015b. The JAK/STAT3 Pathway Is a Common Inducer of Astrocyte Reactivity in Alzheimer's and Huntington's Diseases. J Neurosci 35(6), 2817-2829.

Bienkowski, M.S., Bowman, I., Song, M.Y., Gou, L., Ard, T., Cotter, K., Zhu, M., Benavidez, N.L., Yamashita, S., Abu-Jaber, J., Azam, S., Lo, D., Foster, N.N., Hintiryan, H., Dong, H.W., 2018. Integration of gene expression and brain-wide connectivity reveals the multiscale organization of mouse hippocampal networks. Nat Neurosci 21(11), 1628-1643.

Blanchard, J., Wanka, L., Tung, Y.C., Cardenas-Aguayo Mdel, C., LaFerla, F.M., Iqbal, K., GrundkeIqbal, I., 2010. Pharmacologic reversal of neurogenic and neuroplastic abnormalities and cognitive impairments without affecting Abeta and tau pathologies in 3xTg-AD mice. Acta Neuropathol 120(5), 605-621.

Braak, H., Braak, E., 1991. Neuropathological stageing of Alzheimer-related changes. Acta Neuropathol 82(4), 239-259.

Calhoon, G.G., Tye, K.M., 2015. Resolving the neural circuits of anxiety. Nat Neurosci 18(10), 13941404.

Carroll, J.C., Rosario, E.R., Chang, L., Stanczyk, F.Z., Oddo, S., LaFerla, F.M., Pike, C.J., 2007. Progesterone and estrogen regulate Alzheimer-like neuropathology in female 3xTg-AD mice. J Neurosci 27(48), 13357-13365.

Carroll, J.C., Rosario, E.R., Kreimer, S., Villamagna, A., Gentzschein, E., Stanczyk, F.Z., Pike, C.J., 2010. Sex differences in beta-amyloid accumulation in 3xTg-AD mice: role of neonatal sex steroid hormone exposure. Brain Res 1366, 233-245.

Ceyzériat, K., Abjean, L., Carrillo-de Sauvage, M.A., Ben Haim, L., Escartin, C., 2016. The complex STATes of astrocyte reactivity: How are they controlled by the JAK-STAT3 pathway? Neuroscience 330, 205-218.

Ceyzeriat, K., Ben Haim, L., Denizot, A., Pommier, D., Matos, M., Guillemaud, O., Palomares, M.A., Abjean, L., Petit, F., Gipchtein, P., Gaillard, M.C., Guillermier, M., Bernier, S., Gaudin, M., Auregan, G., Josephine, C., Dechamps, N., Veran, J., Langlais, V., Cambon, K., Bemelmans, A.P., Baijer, J., Bonvento, G., Dhenain, M., Deleuze, J.F., Oliet, S.H.R., Brouillet, E., Hantraye, P., Carrillo-de Sauvage, M.A., Olaso, R., Panatier, A., Escartin, C., 2018. Modulation of astrocyte reactivity improves functional deficits in mouse models of Alzheimer's disease. Acta neuropathologica communications 6(1), 104. 
Chun, H., Lee, C.J., 2018. Reactive astrocytes in Alzheimer's disease: A double-edged sword. Neuroscience research 126, 44-52.

Dabir, D.V., Robinson, M.B., Swanson, E., Zhang, B., Trojanowski, J.Q., Lee, V.M., Forman, M.S., 2006. Impaired glutamate transport in a mouse model of tau pathology in astrocytes. J Neurosci 26(2), 644-654.

De Strooper, B., Karran, E., 2016. The Cellular Phase of Alzheimer's Disease. Cell 164(4), 603-615.

Delekate, A., Fuchtemeier, M., Schumacher, T., Ulbrich, C., Foddis, M., Petzold, G.C., 2014.

Metabotropic P2Y1 receptor signalling mediates astrocytic hyperactivity in vivo in an Alzheimer's disease mouse model. Nature communications 5, 5422.

Escartin, C., Guillemaud, O., Carrillo de Sauvage, M.A., 2019. Questions and (some) answers on reactive astrocytes. Glia in press.

Funahashi, S., 2017. Working Memory in the Prefrontal Cortex. Brain sciences 7(5).

Furman, J.L., Sama, D.M., Gant, J.C., Beckett, T.L., Murphy, M.P., Bachstetter, A.D., Van Eldik, L.J., Norris, C.M., 2012. Targeting astrocytes ameliorates neurologic changes in a mouse model of Alzheimer's disease. J Neurosci 32(46), 16129-16140.

Goni, F., Herline, K., Peyser, D., Wong, K., Ji, Y., Sun, Y., Mehta, P., Wisniewski, T., 2013. Immunomodulation targeting of both Abeta and tau pathological conformers ameliorates Alzheimer's disease pathology in TgSwDI and 3xTg mouse models. J Neuroinflammation 10, 150.

Hamelin, L., Lagarde, J., Dorothee, G., Leroy, C., Labit, M., Comley, R.A., de Souza, L.C., Corne, H., Dauphinot, L., Bertoux, M., Dubois, B., Gervais, P., Colliot, O., Potier, M.C., Bottlaender, M., Sarazin, M., Clinical, I.t., 2016. Early and protective microglial activation in Alzheimer's disease: a prospective study using 18F-DPA-714 PET imaging. Brain 139(Pt 4), 1252-1264.

Hardy, J.A., Higgins, G.A., 1992. Alzheimer's disease: the amyloid cascade hypothesis. Science 256(5054), 184-185.

Heneka, M.T., Carson, M.J., El Khoury, J., Landreth, G.E., Brosseron, F., Feinstein, D.L., Jacobs, A.H., Wyss-Coray, T., Vitorica, J., Ransohoff, R.M., Herrup, K., Frautschy, S.A., Finsen, B., Brown, G.C., Verkhratsky, A., Yamanaka, K., Koistinaho, J., Latz, E., Halle, A., Petzold, G.C., Town, T., Morgan, D., Shinohara, M.L., Perry, V.H., Holmes, C., Bazan, N.G., Brooks, D.J., Hunot, S., Joseph, B., Deigendesch, N., Garaschuk, O., Boddeke, E., Dinarello, C.A., Breitner, J.C., Cole, G.M., Golenbock, D.T., Kummer, M.P., 2015. Neuroinflammation in Alzheimer's disease. Lancet Neurol 14(4), 388-405.

Heneka, M.T., Sastre, M., Dumitrescu-Ozimek, L., Dewachter, I., Walter, J., Klockgether, T., Van Leuven, F., 2005. Focal glial activation coincides with increased BACE1 activation and precedes amyloid plaque deposition in APP[V717I] transgenic mice. J Neuroinflammation 2, 22.

Hirata-Fukae, C., Li, H.F., Hoe, H.S., Gray, A.J., Minami, S.S., Hamada, K., Niikura, T., Hua, F., Tsukagoshi-Nagai, H., Horikoshi-Sakuraba, Y., Mughal, M., Rebeck, G.W., LaFerla, F.M., Mattson, M.P., Iwata, N., Saido, T.C., Klein, W.L., Duff, K.E., Aisen, P.S., Matsuoka, Y., 2008. Females exhibit more extensive amyloid, but not tau, pathology in an Alzheimer transgenic model. Brain Res 1216, 92-103.

Hol, E.M., Pekny, M., 2015. Glial fibrillary acidic protein (GFAP) and the astrocyte intermediate filament system in diseases of the central nervous system. Current opinion in cell biology 32, 121130.

Jo, S., Yarishkin, O., Hwang, Y.J., Chun, Y.E., Park, M., Woo, D.H., Bae, J.Y., Kim, T., Lee, J., Chun, H., Park, H.J., Lee, D.Y., Hong, J., Kim, H.Y., Oh, S.J., Park, S.J., Lee, H., Yoon, B.E., Kim, Y., Jeong, Y., Shim, I., Bae, Y.C., Cho, J., Kowall, N.W., Ryu, H., Hwang, E., Kim, D., Lee, C.J., 2014. GABA from reactive astrocytes impairs memory in mouse models of Alzheimer's disease. Nat Med.

Kahlson, M.A., Colodner, K.J., 2015. Glial Tau Pathology in Tauopathies: Functional Consequences. Journal of experimental neuroscience 9(Suppl 2), 43-50.

Kametani, F., Hasegawa, M., 2018. Reconsideration of Amyloid Hypothesis and Tau Hypothesis in Alzheimer's Disease. Frontiers in neuroscience 12, 25.

Leyns, C.E.G., Holtzman, D.M., 2017. Glial contributions to neurodegeneration in tauopathies. Molecular neurodegeneration 12(1), 50.

Li, S., Jin, M., Liu, L., Dang, Y., Ostaszewski, B.L., Selkoe, D.J., 2018. Decoding the synaptic dysfunction of bioactive human AD brain soluble Abeta to inspire novel therapeutic avenues for Alzheimer's disease. Acta neuropathologica communications 6(1), 121. 
Lian, H., Yang, L., Cole, A., Sun, L., Chiang, A.C., Fowler, S.W., Shim, D.J., Rodriguez-Rivera, J., Taglialatela, G., Jankowsky, J.L., Lu, H.C., Zheng, H., 2015. NFkappaB-Activated Astroglial Release of Complement C3 Compromises Neuronal Morphology and Function Associated with Alzheimer's Disease. Neuron 85(1), 101-115.

Masliah, E., Alford, M., DeTeresa, R., Mallory, M., Hansen, L., 1996. Deficient glutamate transport is associated with neurodegeneration in Alzheimer's disease. Ann Neurol 40(5), 759-766.

Masters, C.L., Bateman, R., Blennow, K., Rowe, C.C., Sperling, R.A., Cummings, J.L., 2015. Alzheimer's disease. Nature reviews. Disease primers 1, 15056.

Medina, M., Avila, J., 2014. New perspectives on the role of tau in Alzheimer's disease. Implications for therapy. Biochemical pharmacology 88(4), 540-547.

Oddo, S., Caccamo, A., Shepherd, J.D., Murphy, M.P., Golde, T.E., Kayed, R., Metherate, R., Mattson, M.P., Akbari, Y., LaFerla, F.M., 2003. Triple-transgenic model of Alzheimer's disease with plaques and tangles: intracellular Abeta and synaptic dysfunction. Neuron 39(3), 409-421.

Querfurth, H.W., LaFerla, F.M., 2010. Alzheimer's disease. N Engl J Med 362(4), 329-344.

Reichenbach, N., Delekate, A., Plescher, M., Schmitt, F., Krauss, S., Blank, N., Halle, A., Petzold, G.C., 2019. Inhibition of Stat3-mediated astrogliosis ameliorates pathology in an Alzheimer's disease model. EMBO molecular medicine 11(2).

Ries, M., Sastre, M., 2016. Mechanisms of Abeta Clearance and Degradation by Glial Cells. Frontiers in aging neuroscience 8,160 .

Sala Frigerio, C., De Strooper, B., 2016. Alzheimer's Disease Mechanisms and Emerging Roads to Novel Therapeutics. Annual review of neuroscience 39, 57-79.

Sancheti, H., Patil, I., Kanamori, K., Diaz Brinton, R., Zhang, W., Lin, A.L., Cadenas, E., 2014. Hypermetabolic state in the 7-month-old triple transgenic mouse model of Alzheimer's disease and the effect of lipoic acid: a 13C-NMR study. J Cereb Blood Flow Metab 34(11), 1749-1760.

Serrano-Pozo, A., Frosch, M.P., Masliah, E., Hyman, B.T., 2011. Neuropathological alterations in Alzheimer disease. Cold Spring Harbor perspectives in medicine 1(1), a006189.

Sompol, P., Furman, J.L., Pleiss, M.M., Kraner, S.D., Artiushin, I.A., Batten, S.R., Quintero, J.E., Simmerman, L.A., Beckett, T.L., Lovell, M.A., Murphy, M.P., Gerhardt, G.A., Norris, C.M., 2017. Calcineurin/NFAT Signaling in Activated Astrocytes Drives Network Hyperexcitability in AbetaBearing Mice. J Neurosci 37(25), 6132-6148.

Verkhratsky, A., Nedergaard, M., 2018. Physiology of Astroglia. Physiological reviews 98(1), 239-389.

West, M.J., Gundersen, H.J., 1990. Unbiased stereological estimation of the number of neurons in the human hippocampus. J Comp Neurol 296(1), 1-22.

Wu, Z., Guo, Z., Gearing, M., Chen, G., 2014. Tonic inhibition in dentate gyrus impairs long-term potentiation and memory in an Alzhiemer's disease model. Nature communications 5, 4159. 
Click here to download high resolution image

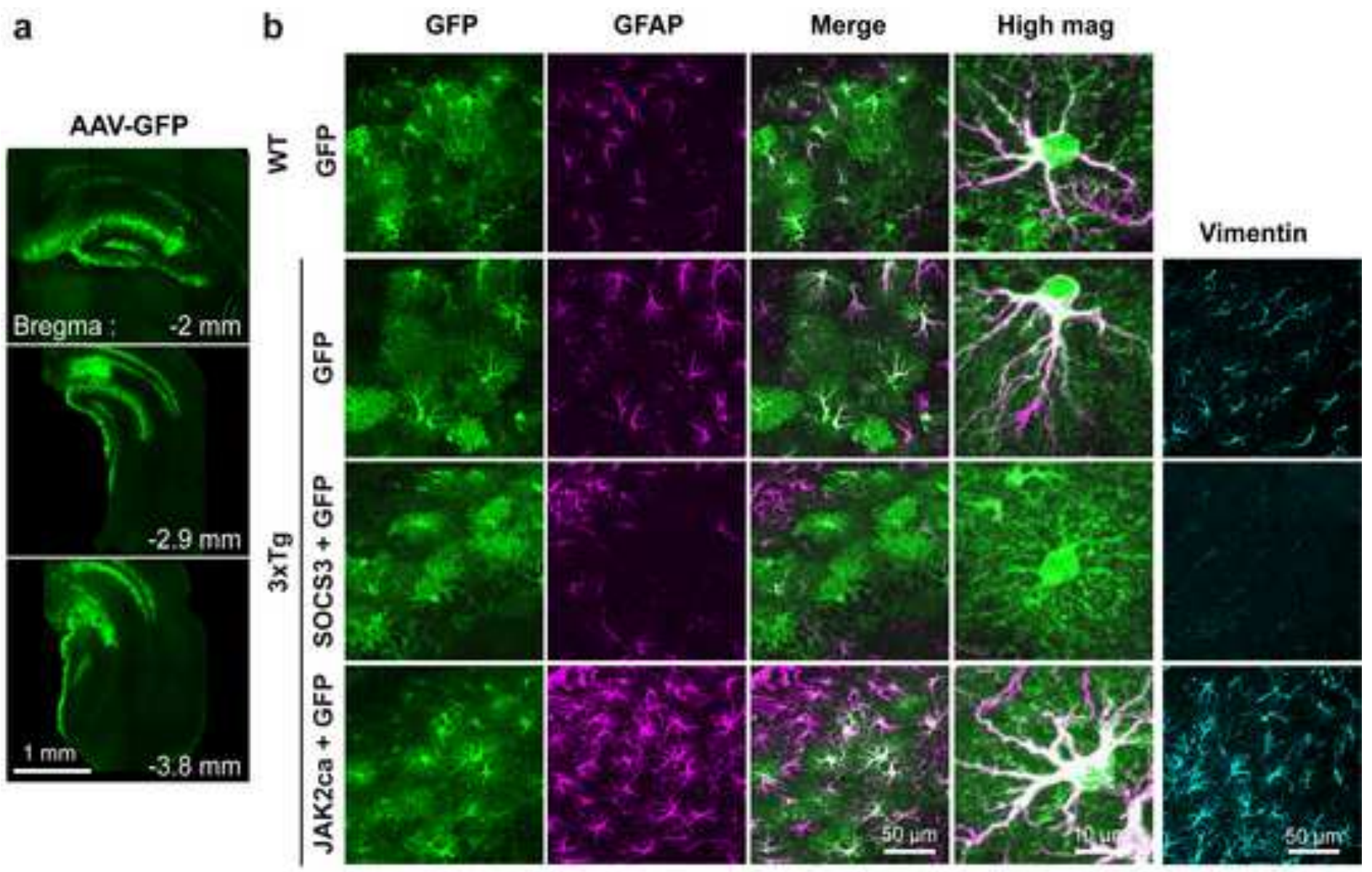

\section{$\square$ WT-GFP $\square 3 \times T g$-GFP $\square 3 \times T g$-SOCS3 $\square 3 \times T g$ JAK2Ca}

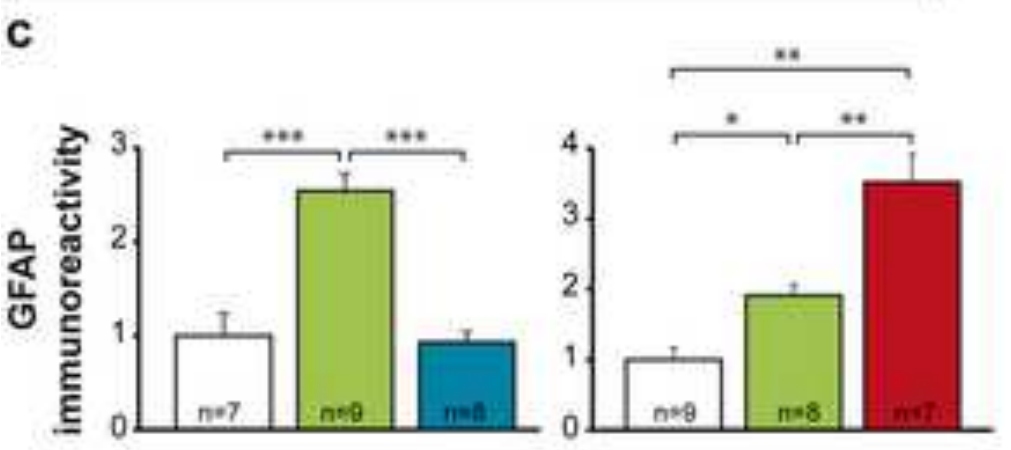

d

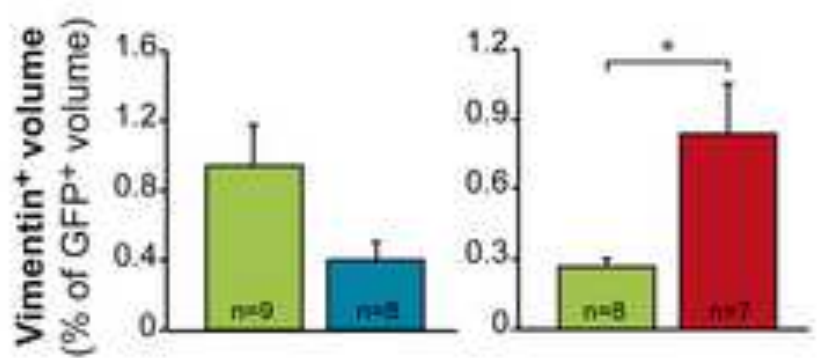

e
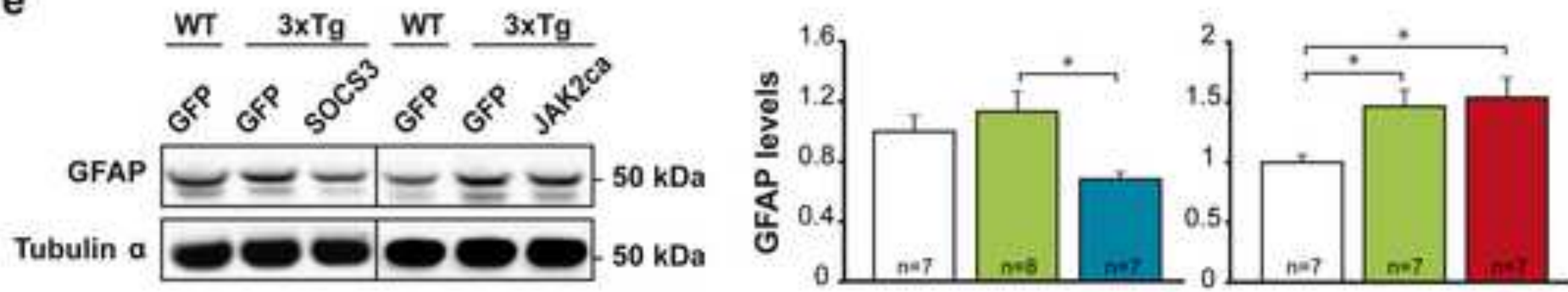
Figure2
Click here to download high resolution image

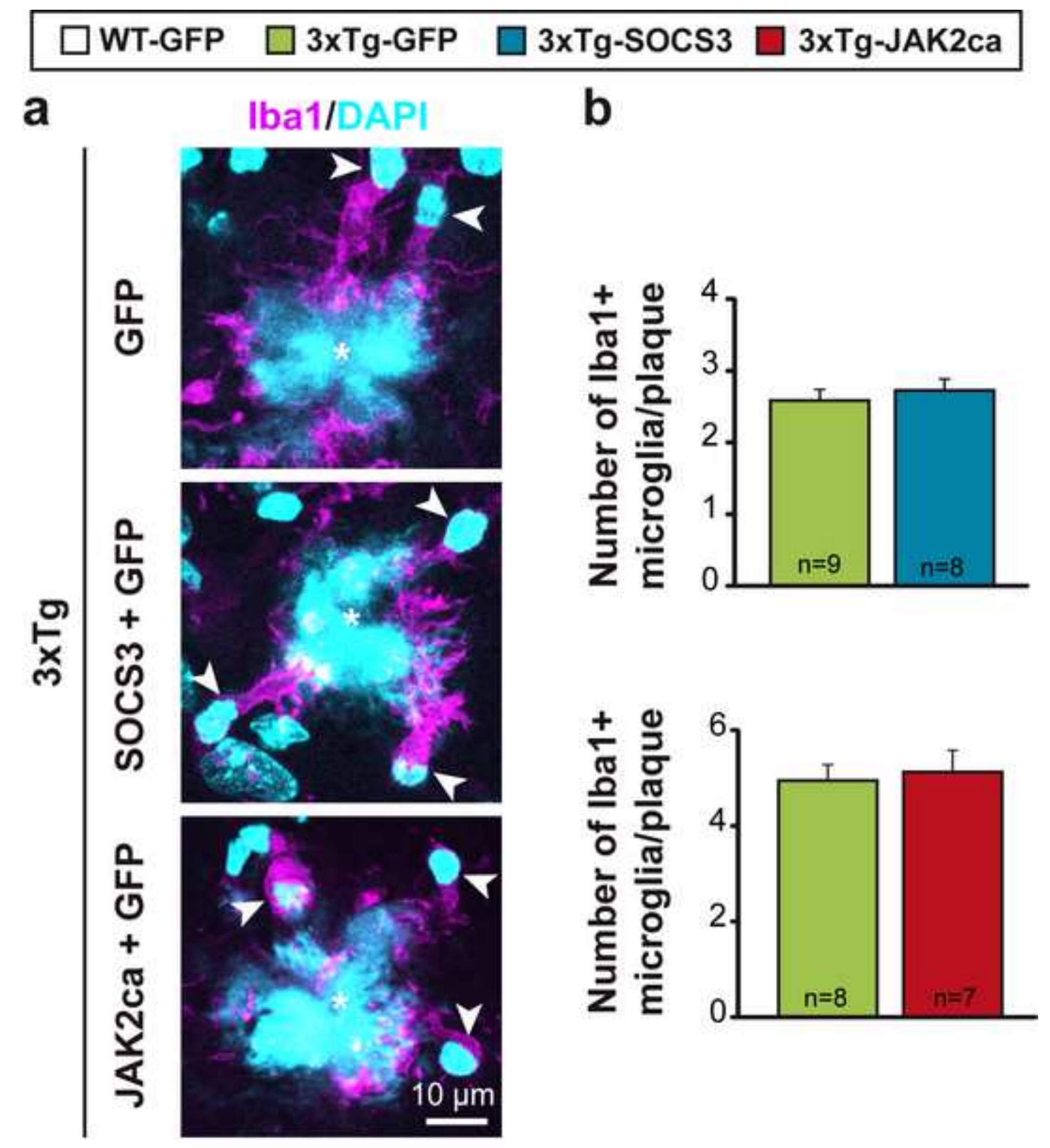


a
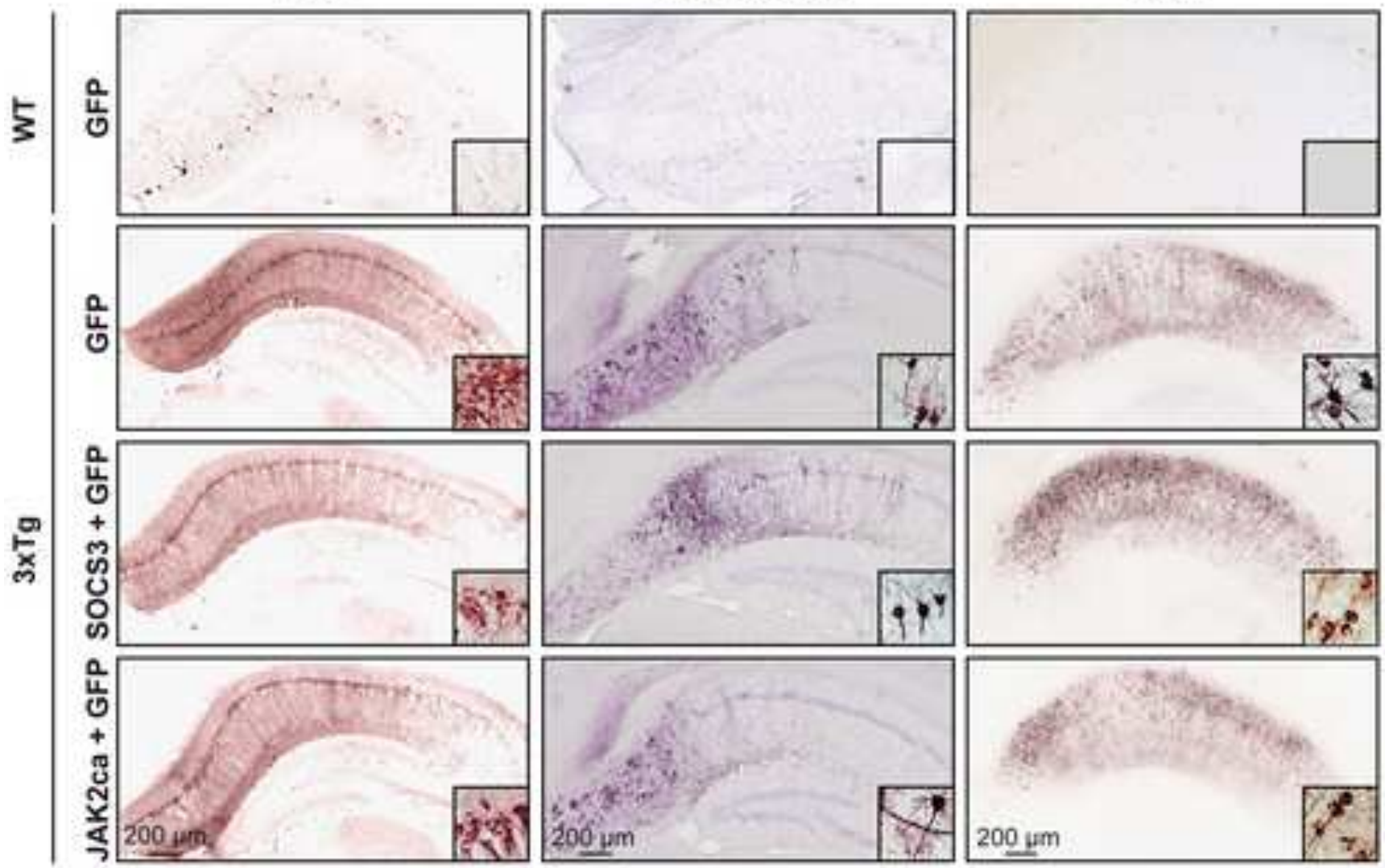

b
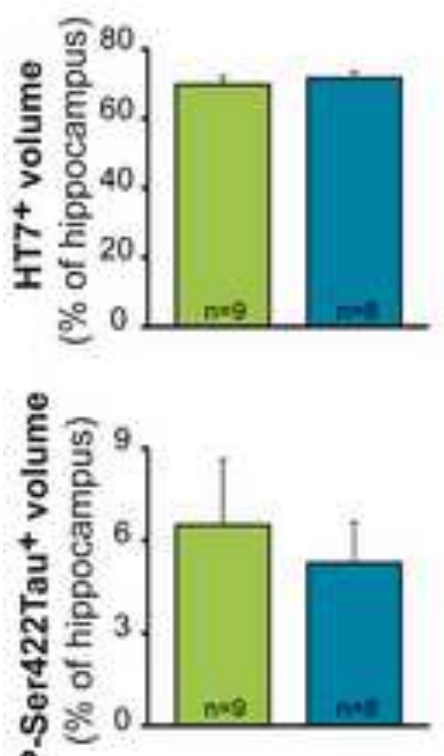

0

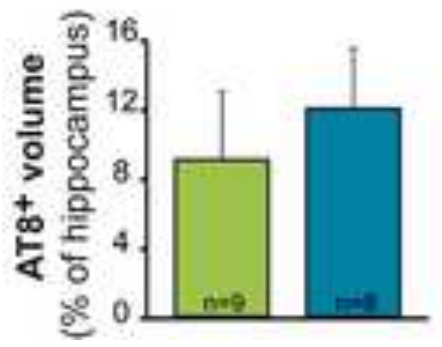

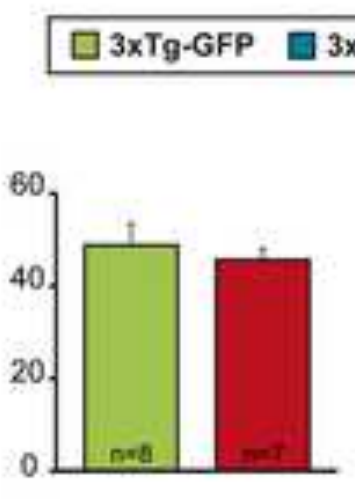

C

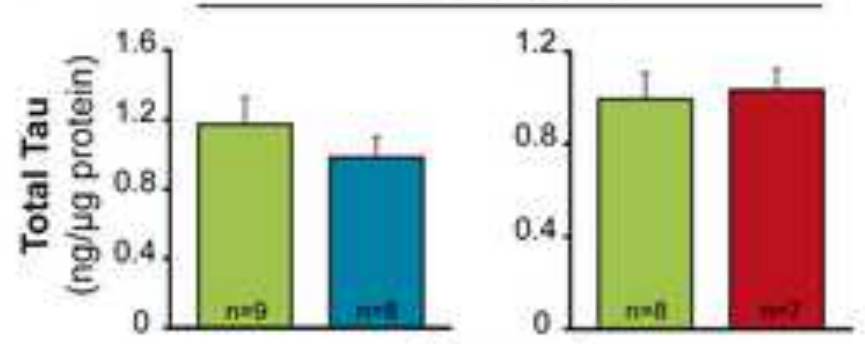

16 months
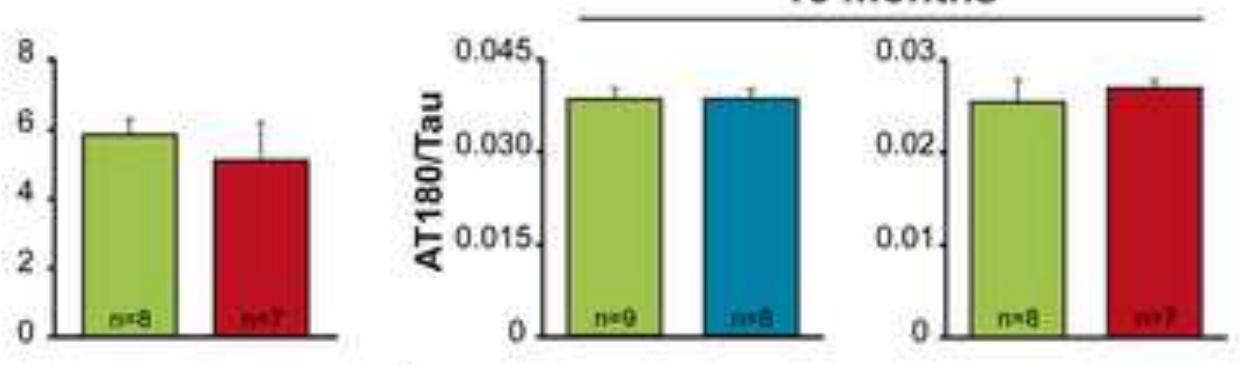

d

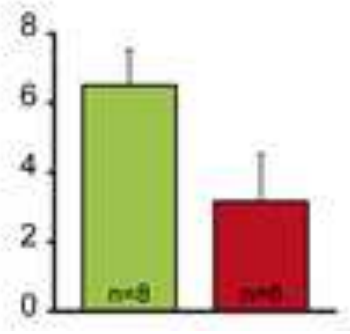

\section{6 months}

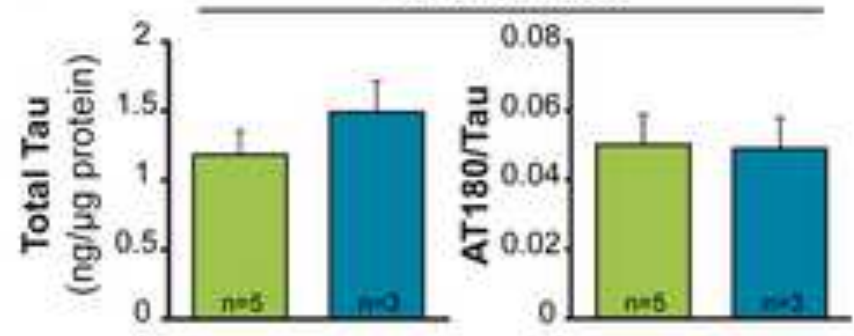


Click here to download high resolution image
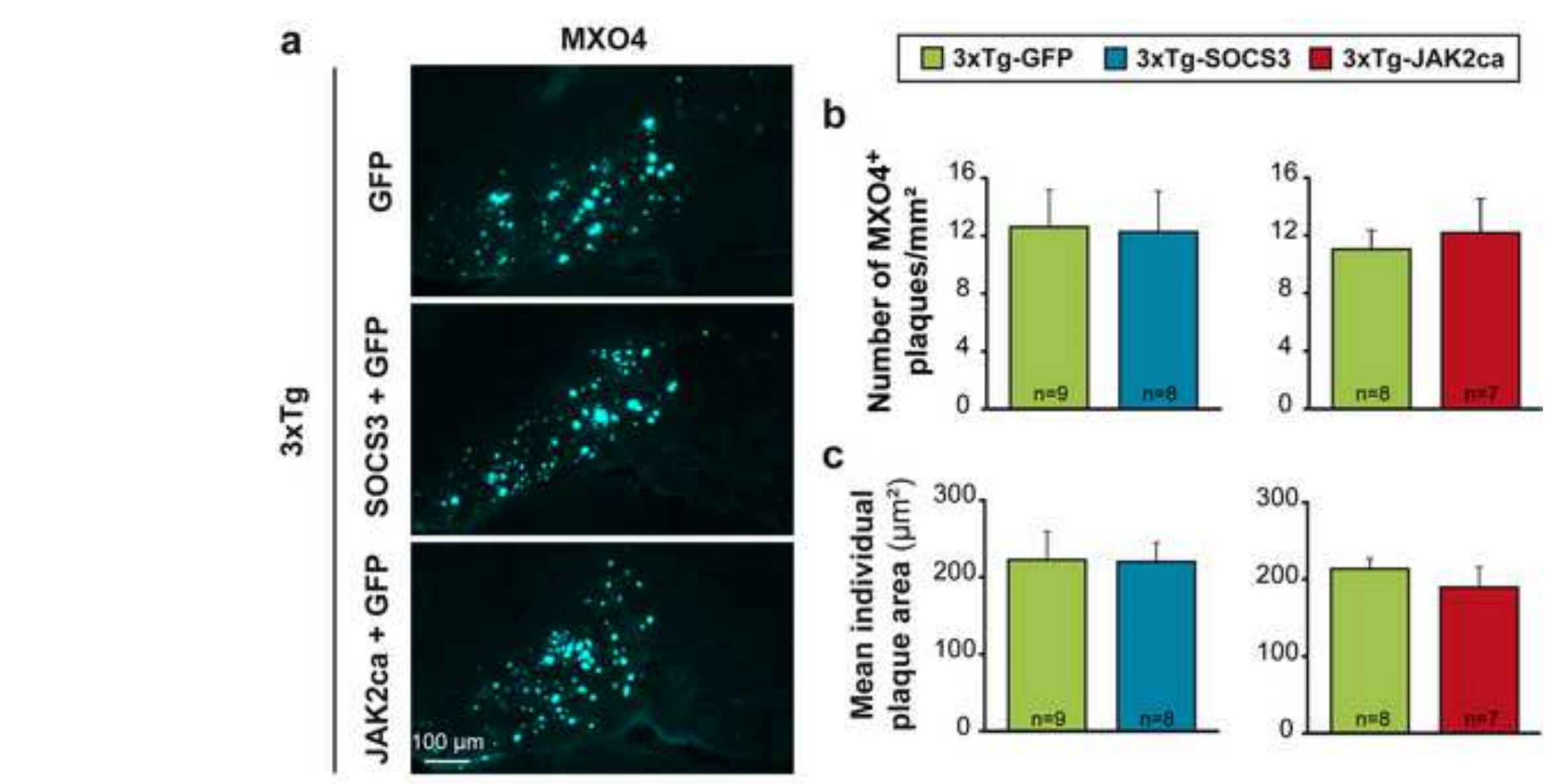

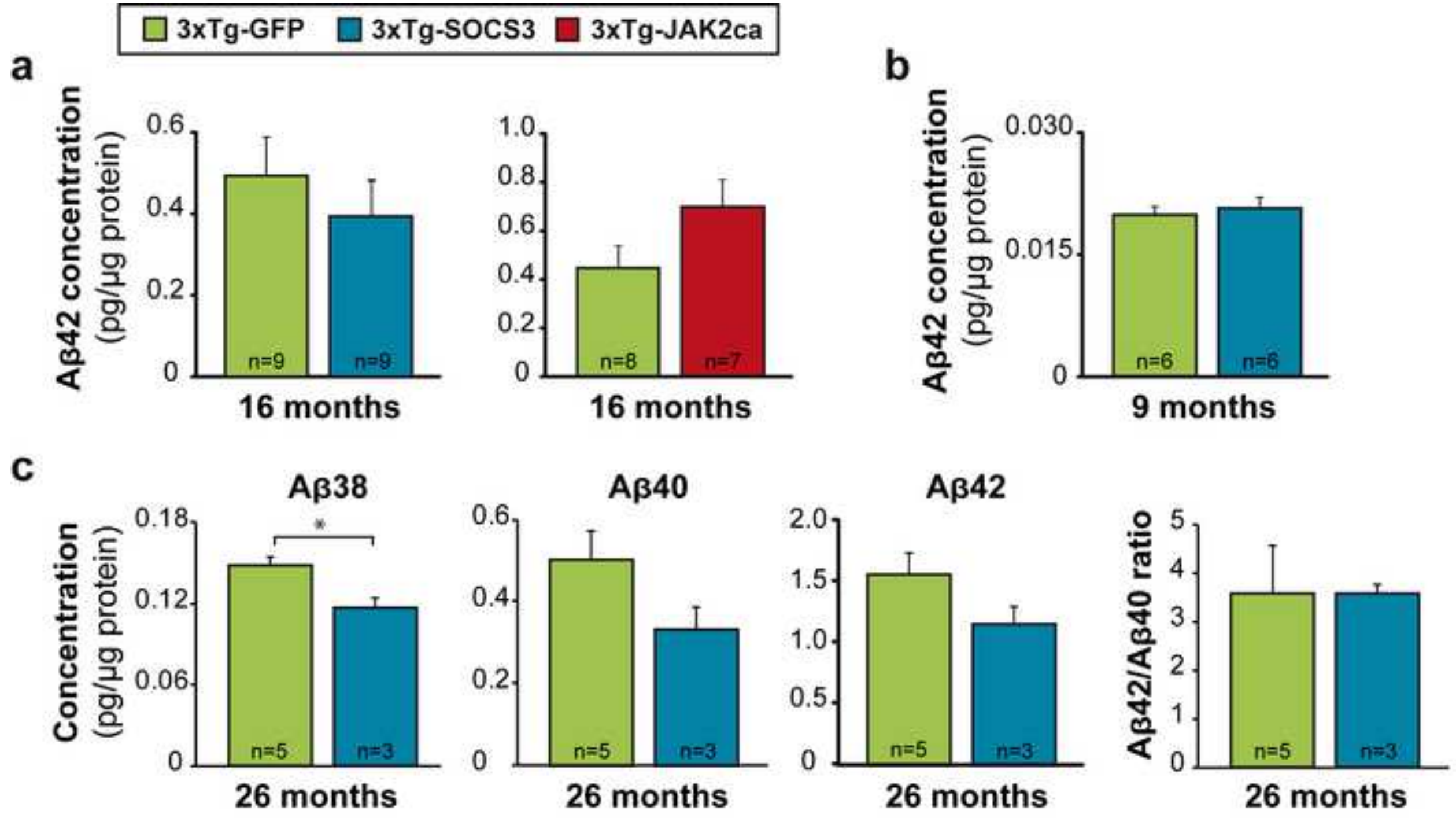


\section{DWT-GFP $\square 3 \times T_{\text {g }}$-GFP $\square 3 \times \mathrm{Tg}$-SOCS 3}

a
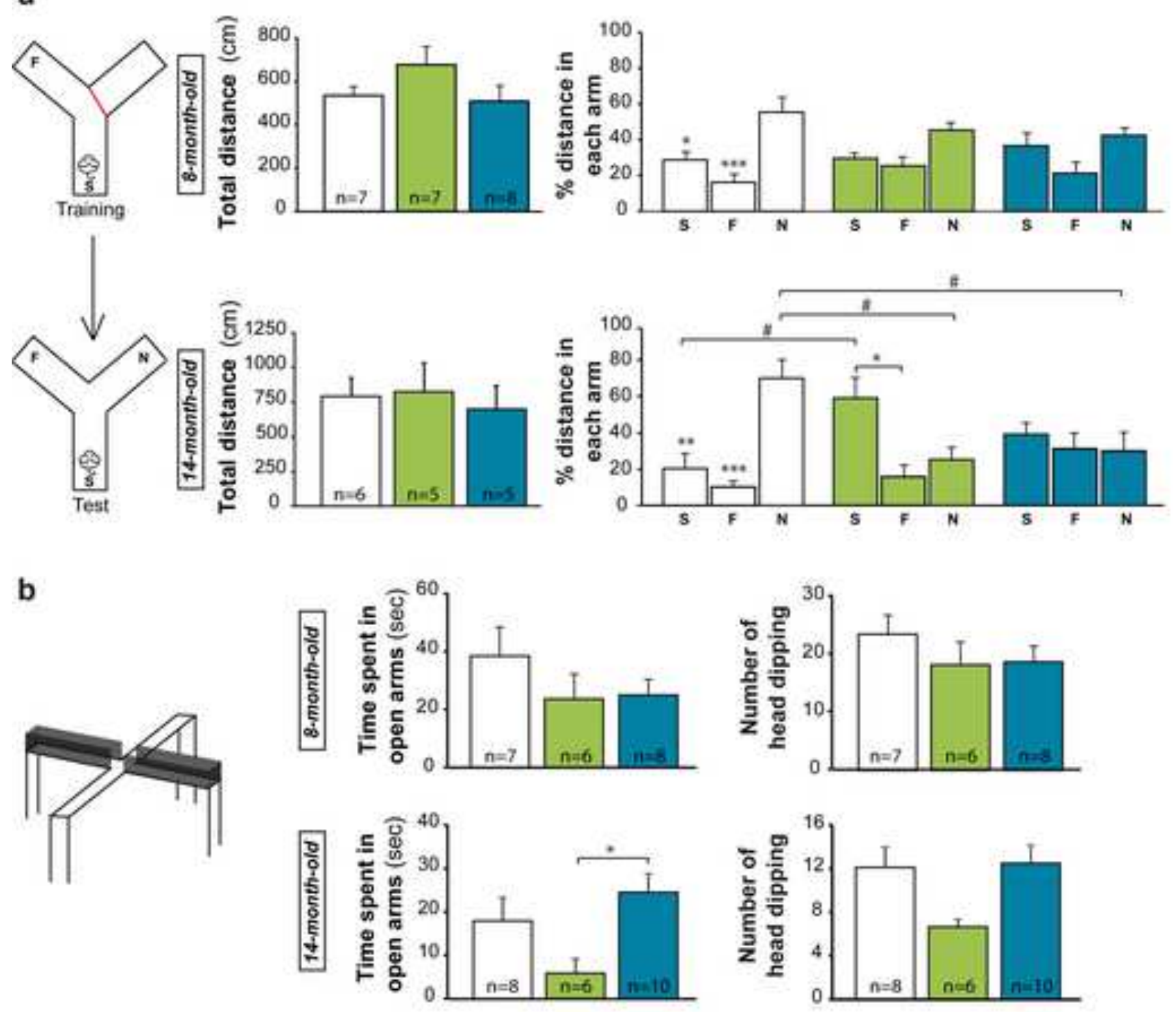
Supplemental Fig1
Click here to downl

Click here to download Supplemental or Multimedia Files: Sup1-Timeline.tif

Click here to download Supplemental or Multimedia Files: Sup1-Timeline.tif 

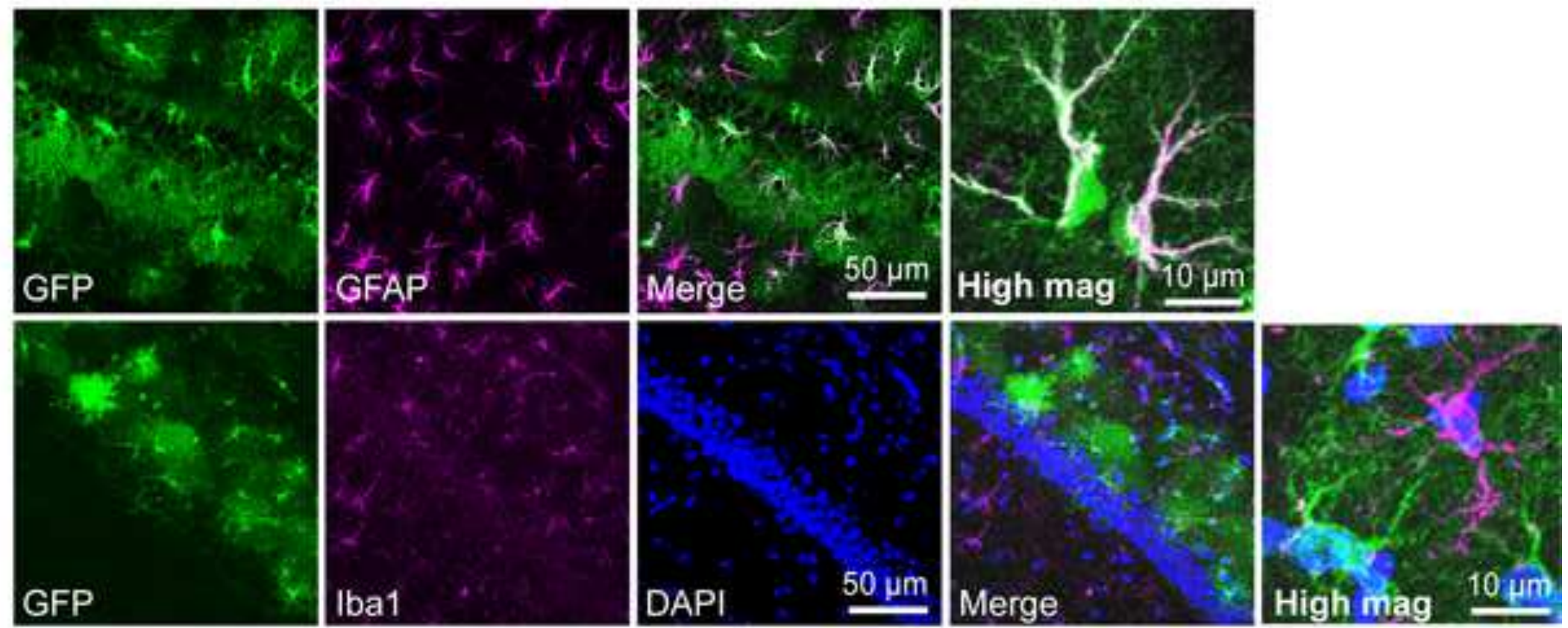
Supplemental Fig3
Click here to downl

Click here to download Supplemental or Multimedia Files: Supp3-3xTg-WB-P-Tau-v10.tif 
Supplemental Fig4
Click here to download Supplemental or Multimedia Files: Supp4-3xTg-Ab42-4G8-v10.tif

Supplemental Fig4
Click here to download Supplemental or Multimedia Files: Supp4-3xTg-Ab42-4G8-v10.tif Click here to download Supplemental or Multimedia Files: Supp4-3xTg-Ab42-4G8-v10.tif

(1)

.

(1)

(1)

(1) (1) (1) . . . . . . . . . . . . . . . . 
Supplemental Fig5
Click here to download Supplemental or Multimedia Files: Supp-5-3xTg-WB-v10.tif

Supplemental Fig5
Click here to download Supplemental or Multimedia Files: Supp-5-3xTg-WB-v10.tif 更

r

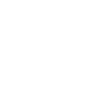

(2)

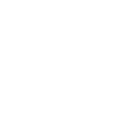

.

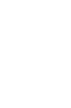




\section{Credit Author Statement}

KCe, OG: main experimentations \& analysis, writing \& editing. TSG: experimentation (HT7 \& A $\beta 42$ quantification), KC, ASH: methodology (behavioral analysis; image scanning and analysis, respectively). FP, MACS: experimentation (histology and western blotting, respectively). MG, LBH, SB: experimentation (injections). CJ, AB: methodology (viral vector production); EB, PH, GB: resources. CE: conceptualization, data curation, project supervision, funding acquisition, writing original draft \& editing. All authors reviewed the manuscript. 